УДК 662.638

\title{
КИНЕТИЧЕСКИЕ ХАРАКТЕРИСТИКИ ПИРОЛИЗА БИОМАССЫ
}

\author{
Табакаев Роман Борисович1, \\ TabakaevRB@tpu.ru
}

\section{Алтынбаева Дарига Бахитжановна', dba10@tpu.ru}

\author{
Ибраева Канипа Талгатовна', \\ kti1@tpu.ru
}

\author{
Заворин Александр Сергеевич1, \\ zavorin@tpu.ru \\ 1 Национальный исследовательский Томский политехнический университет,
Россия, 634050, г. Томск, пр. Ленина, 30.
}

\begin{abstract}
Актуальность исследования обусловлена тенденцией увеличения доли использования возобновляемых источников энергии в топливно-энергетическом балансе для снижения вредного воздействия на окружающую среду.

Цель: определение кинетических параметров пиролиза биомассы методом Г. Фридмана на основе результатов дифрференциального термического анализа.
\end{abstract}

объекты: солома, отходы зернопереработки (пшеничные отруби), скорлупа кедровых орехов, сосновые опилки, низинный торфф Суховского месторождения, а также отходы жизнедеятельности крупнорогатого скота.

Методы. Теплотехнические характеристики образцов биомассы определены согласно аттестованным методикам (ГОСТ 11305-2013, ГОСТ 11306-2013, ГОСТ Р 54186-2010, ГОСТ Р 56881-2016, ГОСТ 32990-2014); значения теплоты сгорания установлены при помощи калориметра АБК-1 (РЭТ, Россия) в соответствии с ГОСТ 147-2013 (ISO 1928-2009); элементный анализ её органической части проведен на приборе VarioMicroCube (Elementar, Германия); кинетические характеристики низкотемпературного пиролиза биомассы определены методом Г. Фридмана на основе диффференциального термического анализа, проведенного при температуре 313-1273 К при скорости нагрева 5, 15 и 30 K/мин.

Результаты. Термическое разложение биомассы в интервале 313-1273 K происходит в две стадии: первая стадия протекает в интервале температур от 463-488 до 623-653 K и характеризуется резким снижением массы образцов от 24 (торф) до 63 \% (опилки); вторая стадия наблюдается от 623-653 до 873 К со значительно меньшим изменением массы образцов (от 9 до 14 \%). При дальнейшем увеличении температуры органическая часть образцов практически не претерпела преобразования, изменения произошли только в минеральной части торфа при температуре 923-1123 К и обусловлены разложением карбоната кальция. Определены зависимости энергии активации ( $\left.E_{a}\right)$ om степени конверсии биомассы (w/wo), по которым рассчитаны её средние значения: для соломы $\left(E a_{\mathrm{cp}}^{\mathrm{co}}\right)-21,4$ кДж моль-1; для опилок $\left(E a_{\mathrm{cp}}^{\text {oП }}\right)-20,7$ кДж моль-1; для скорлупы $\left(E a_{\mathrm{cp}}^{\mathrm{c \kappa}}\right)-24,2$ кДж моль ${ }^{-1}$; для отходов жизнедеятельности крупнорогатого скота $\left(E a_{\mathrm{cp}}^{\mathrm{H}}\right)-23,1$ кДж моль ${ }^{-1}$; для отрубей $\left(E a_{\mathrm{cp}}^{\text {oт }}\right)-33,1$ кДж моль- ${ }^{-1}$ для торфра $\left(E a_{\mathrm{cp}}^{\mathrm{T}}\right)-24,0$ кДж моль ${ }^{-1}$. Значения предэкспоненциального множителя (A) для исследуемых видов биомассы находятся в диапазоне 82,42-2377,01 час-1.

\section{Ключевые слова:}

Биомасса, термическая переработка, диффреренциальный термический анализ, кинетика, метод Фридмана.

\section{Введение}

Традиционная энергетика, основанная на сжигании ископаемых органических топлив (угля, газа и нефтяных продуктов), является одним из основных источников загрязнения окружающей среды и изменения климата планеты $[1,2]$. Техногенное воздействие энергетики на окружающую среду приводит к существенному ухудшению здоровья и преждевременной смертности населения [3]. Учитывая прогнозируемый рост населения планеты [4-6], неизбежно сопровождающийся ростом энергопотребления [7], мировое энергетическое сообщество стремится к переходу на экологически чистую энергетику, повышению энерго- и ресурсоэффективности природопользования.

В связи с этим во многих странах особое внимание уделяется использованию возобновляемых источников энергии (ВИЭ) [8-12]. Согласно данным Международного энергетического агентства доля энергии, выработанной на основе ВИЭ, в мировом масштабе составляет 11,5 \% (без учета гидроэнергетики) [13]. При этом некоторыми странами даже рассматриваются сценарии [8, 14-18] по полному переводу энергетики на ВИЭ в обозримом будущем.

Согласно [19] среди различных видов ВИЭ в топливно-энергетическом балансе значительная доля использования отводится биомассе. Она является наиболее распространенным источником среди прочих видов ВИЭ [20, 21], что делает её универсальным ресурсом для повсеместного использования. Актуальность вовлечения в энергетику для биомассы обусловлена также тем, что она по большей части представлена отходами различных сфер деятельности (опилки, лузга, солома, отходы зернопереработки и т. п.) или продуктами разложения органики (торф, ил водоемов). Оставаясь неиспользованными, эти ресурсы могут нанести вред окружающей среде или создать потенциальную опасность возгорания, приводя к природным и антропогенным пожарам [22-25]. 
Традиционные технологии сжигания органических топлив, успешно применяемые для угля, зачастую оказываются неэффективными при использовании биомассы. Это обусловлено её специфическими характеристиками. В исходном состоянии биомасса имеет высокую влажность (30,4-79,7 \% [26]), что существенно осложняет процесс транспортировки при низких температурах окружающего воздуха (смерзается в зимний период) [27] и требует при сжигании высоких эксплуатационных затрат на испарение влаги. Несмотря на низкое значение зольности биомассы, её минеральная часть в процессе горения приводит к шлакованию поверхностей нагрева [28, 29]. Мелкий фракционный состав (например, при рассмотрении опилок или соломы) и крошимость не позволяют сжигать биомассу в слоевых топочных устройствах без их модернизации.

В связи с вышеописанным необходимо искать альтернативные пути, позволяющие вовлечь ресурсы биомассы в мировой топливно-энергетический баланс. Одним из перспективных направлений, позволяющих перерабатывать биомассу в химически и энергетически ценные продукты, является пиролиз. Пиролиз представляет собой многостадийный процесс термического разложения органического сырья при отсутствии кислорода. В процессе пиролиза образуются твердый углеродистый остаток, жидкие продукты (пирогенетическая вода и смола) и горючий газ, состав и характеристики которых существенно зависят от природы исходного сырья, температурного режима давления, скорости нагрева сырья и длительности его переработки. Столь сложная зависимость, учитывающая большое количество параметров, и многообразность протекающих при этом химических реакций приводят к тому, что наиболее распространенным методом исследования пиролиза всё ещё остается физический эксперимент [30-34]. Однако ведутся исследования по разработке алгоритмов численных методов изучения и моделированию процессов термического разложения биомассы, способных существенно снизить временные и трудовые затраты при проектировании оборудования, его наладке и внедрении в технологический процесс. Реализация этой задачи требует подробного изучения кинетики происходящих при пиролизе процессов.

В теории химической кинетики широко известны два подхода к определению кинетических характеристик (энергии активации и предэкспоненциального множителя) процессов химического реагирования [35 36]: экспериментальные методы без построения модели и методы подбора моделей. Последние методы являются достаточно трудоемкими, т. к. для достоверного описания реальных процессов необходимо использовать сложный математический аппарат и соответствующие программные комплексы, например, при проведении квантохимических расчетов [37]. В случае упрощения этих методов необходимо обосновывать большое количество допущений. Например, обычно считается, что плавное изменение параметров процесса химического реагирования не приводит к резкому изменению кинетических характеристик, что в реальных условиях не всегда выполняется. Поэтому для оценки достоверности полученных кинетических характеристик необходимо воспроизведение натурных условий эксперимента.

Методы без построения модели (методы Фридмана, Коутса и Редферна, Киссинджера-АкахирыСуносе, Озавы-Флинна-Уолла и др.) являются менее трудоемкими и более надежными [38-41], поэтому они более широко используются для вычисления кинетических характеристик процессов по данным об изменении массы образцов (термогравиметрического анализа - ТГ) в процессе термического разложения. Получаемые при расчете кинетики характеристики являются в дальнейшем исходными данными для разработки прогностических математических моделей и проведения численного моделирования соответствующих физико-химических процессов в широких диапазонах варьирования параметров исследуемой системы и внешних воздействий.

Исследование кинетических параметров биомассы встречается во многих работах, например, в [42-45]. Однако необходимо отметить, что согласно [46] характеристики биомассы демонстрируют большую вариативность даже в зависимости от её вида, типа растительности, стадии роста и условий выращивания. Эти свойства обычно оказывают большое влияние на скорость сгорания биомассы, способ сжигания, выбросы и общую эффективность сжигания, а соответственно и на кинетические параметры. Это приводит к необходимости изучения кинетики для каждого отдельного вида биомассы применительно к конкретному региону её происхождения.

В настоящей работе рассматриваются ресурсы биомассы Томской области, являющейся, как и многие другие регионы в Российской Федерации, зависимой от поставок угля с крупных угольных месторождений (Кузбасса или Красноярского края) [47]. При этом область имеет собственные, неиспользуемые на данный момент запасы биоресурсов - торфа, древесины, отходов агропромышленного комплекса [47-49], для энерготехнологического использования которых необходимо изучение кинетических параметров пиролиза. В связи с вышеописанным, целью настоящей работы является определение кинетических параметров пиролиза биомассы Томской области методом Г. Фридмана на основе результатов дифференциального термического анализа.

\section{Методика исследования}

Объект исследования

В качестве исходного сырья для исследований рассмотрено несколько видов биомассы различного происхождения: солома, отходы зернопереработки (пшеничные отруби), скорлупа кедровых орехов, сосновые опилки, низинный торф Суховского месторождения, а также отходы жизнедеятельности крупнорогатого скота (ОЖКС). Предварительно пробы высушены до воздушно-сухого состояния.

Влажность и зольность торфа определяли по соответствующим стандартам ГОСТ 11305-2013 и ГОСТ 11306-2013, для остальных видов биомассы исполь- 
зованы ГОСТ Р 54186-2010 (ЕN 14774-1: 2009) и ГОСТ Р 56881-2016 (Е1755-01). Выход летучих веществ для всех видов биомассы устанавливали согласно методике ГОСТ 32990-2014 (EN 15148: 2009). Теплоту сгорания биомассы определяли при помощи калориметра АБК-1 (РЭТ, Россия) в соответствии с ГОСТ 147-2013 (ISO 1928-2009). Элементный анализ её органической части проводили на приборе VarioMicroCube (Elementar, Германия).

Дифференциальный термический

и гравиметрический анализы

Перед анализом каждую пробу высушивали в лаборатории до достижения воздушно-сухого состояния, после чего измельчали на планетарной мельнице «Pulverisette 6» (FRITSCH, Германия) до пылевидного состояния (размер фракций менее 200 мкм).

Для исследования пиролиза биомассы и расчета его кинетических параметров проведены термогравиметрический анализ (ТГ) и дифференциальная сканирующая калориметрия (ДСК) на синхронном термоанализаторе STA 449 F5 Jupiter (Netzsch, Германия). Параметры проведения анализа включали: масса каждой пробы составила 20 мг, скорость нагрева образцов - 5, 15 и $30 \mathrm{~K} /$ мин., температурный интервал нагрева - 313-1273 К. Анализы выполнены в инертной среде аргона (расход 20 мл в минуту) с целью имитации условий осуществления пиролиза.

Данные ТГ использованы для определения кинетических характеристик процесса пиролитического разложения биомассы методом Генри Фридмана [39].

\section{Кинетика термического разложения}

Согласно рекомендациям Международной конфедерации термического анализа и калориметрии (ICTAC) [50] установление зависимости энергии активации $\left(E_{a}\right)$ от степени конверсии образца $\left(w / w_{0}\right)$ является достаточным условием для кинетического описания процесса. В связи с этим для расчета кинетики процесса применен метод Г. Фридмана.

Изоконверсионный метод Фридмана используется для описания стадий процесса химического реагирования, в которых степень превращения $\left(w / w_{0}\right)$ экспериментального образца одинакова [39]. Поэтому для оценки кинетических характеристик процесса горения методом Фридмана необходимы данные дифференциального термического анализа, полученные при нескольких скоростях нагрева образцов. Однако одна и та же степень превращения $\left(w / w_{0}\right)$ достигается при разной температуре в зависимости от скорости нагрева. В [51-54] показано, что данные, полученные по методу Фридмана, имеют хорошую сходимость с результатами физического эксперимента.

Этот метод основан на взаимном сопоставлении результатов экспериментов, проведенных при разных скоростях нагрева образцов [39]. Метод Фридмана позволяет определить энергию активации процессов без построения модели их протекания. Уравнение, описывающее связь энергии активации и предэкспоненциального множителя, имеет вид [39]:

$$
\ln \left(-\frac{1}{w_{0}} \frac{d w}{d t}\right)=\ln A+\ln \left[f\left(\frac{w}{w_{0}}\right)\right]-\frac{E}{R T} .
$$

Отношения энергии активации к универсальной газовой постоянной $(-E / R)$ определяются по наклону кривых, описывающих зависимости $\left(\ln \left(\left(-1 / w_{0}\right)(d w / d t)\right)\right)$ от $(1 / T)$ для группы значений степени превращения топлива $\left(w / w_{0}\right)$. Произведения предэкспоненциального множителя и функции изменения массы образца $\left(\ln \left(A f\left(w / w_{0}\right)\right)\right)$ получают для каждого значения $\left(w / w_{0}\right)$ в точках пересечения с осью ординат.

Далее согласно методу Фридмана принимается, что:

$$
f\left(w / w_{0}\right)=\left[\left(w-w_{f}\right) / w_{0}\right]^{n},
$$

где $w_{f}$ - масса образца при окончании процесса термического разложения, кг; $n-$ кинетический порядок реакции, $\left(w-w_{f}\right)$ - масса топлива, доступная для разложения в любой момент времени, кг.

В результате преобразований уравнений (1) и (2) Фридманом [39] получено:

$$
\ln \left[A f\left(\frac{w}{w_{0}}\right)\right]=\ln \mathrm{A}+n \ln \left[\frac{\left(w-w_{f}\right)}{w_{0}}\right] .
$$

Таким образом, согласно Фридману, получим, что график зависимости $\left(\ln \left(A f\left(w / w_{0}\right)\right)\right)$ от $\ln \left(\left(w-w_{f}\right) / w_{0}\right)$ дает прямую линию, тангенс угла наклона которой равен $n$, а точка пересечения с ось ординат $\ln \left(A f\left(w / w_{0}\right)\right)$.

\section{Исследование состава минеральной части}

В процессе нагрева минеральная часть топлива может претерпевать изменения, сопровождающиеся потерей массы (например, при дегидрации монтмориллонита или разложения известняка), что отразится на результатах ТГ и приведет к неточности расчета кинетики. В связи с этим необходимо предваряющее исследование состава минеральной части биомассы для оценки её влияния на результаты ТГ.

С этой целью проведена рентгеновская дифрактометрия (XRD) рассматриваемых проб с использованием дифрактометра Shimadzu XRD7000 (CuKaизлучения) с счетчиком монохроматора Shimadzu CM-3121. Качественный рентгеновский анализ проводился с использованием базы данных PDF2+. При этом влажность анализируемых образцов соответствовала воздушно-сухому состоянию.

\section{Обсуждение результатов}

Теплотехнические характеристики биомассы

Теплотехнические характеристики исследуемой биомассы представлены в табл. 1. Видно, что зольность рассматриваемых проб представлена в широком диапазоне: от 1,0 \% для скорлупы кедровых орехов до 22,8 \% для суховского торфа. Зольность образцов соломы, отрубей, опилок и скорлупы ниже среднего значения для травянистой и сельскохозяйственной биомассы $\left(A^{d}=8,0 \%\right)$ [55]. Пробы ОЖКС и суховского торфа по величине зольности можно отнести к более минерализованным видам биомассы. Низшая теплота сгорания при достижении пробами воздушно-сухого состояния составляет 11,8-18,1 МДж/кг. 
При рассмотрении суховского торфа, ОЖКС и пшеничных отрубей в качестве исходного сырья для изготовления топливных гранул можно прийти к выводу, что даже в высушенном состоянии низкое значение теплоты сгорания не позволит изготовить пеллеты и брикеты с характеристиками, удовлетворяющими требованиям стандартов DIN и DINplus (более $Q_{i}^{r}=17,5$ и 18 МДж/кг, соответственно) $[56,57]$.

Значительный выход летучих веществ из биомассы $(69,7-83,4 \%)$ свидетельствует о термической нестабильности ее органической массы и высокой реакционной способности при сжигании. Исследуемое сырье обладает низким содержанием серы (не более $0,07 \%$ в пересчете на сухую беззольную массу), что обеспечивает низкое значение количества выбросов оксидов серы (SOx) при его сжигании даже при отсутствии сероочистки дымовых газов.

При помощи рентгенофазового анализа исследован состав минеральной части рассматриваемых проб. Установлено, что минеральная составляющая суховского торфа в основной своей массе представлена карбонатом кальция $\mathrm{CaCO}_{3}$ и оксидом кремния $\mathrm{SiO}_{2}$ (рис. 1). Изучить минералогический состав остальных проб не представилось возможным из-за возникающего в процессе анализа диффузионного гало, наличие которого обусловлено отсутствием кристаллического строения минерализованных компонентов.

Таблица 1. Теплотехнические характеристики исследуемой биомассы

Table 1. Thermotechnical characteristics of biomass

\begin{tabular}{|c|c|c|c|c|c|c|}
\hline $\begin{array}{l}\text { Теплотехническая характеристика } \\
\text { Thermotechnical characteristics }\end{array}$ & $\begin{array}{l}\text { солома } \\
\text { straw }\end{array}$ & $\begin{array}{c}\text { отруби } \\
\text { bran }\end{array}$ & $\begin{array}{c}\text { скорлупа } \\
\text { shell }\end{array}$ & $\begin{array}{l}\text { опилки } \\
\text { sawdust }\end{array}$ & $\begin{array}{c}\text { ОЖКС } \\
\text { waste from cattle }\end{array}$ & $\begin{array}{l}\text { Top中* } \\
\text { peat* }\end{array}$ \\
\hline $\begin{array}{l}\text { Влажность } W^{a}, \% \\
\text { Humidity } W^{a}, \%\end{array}$ & 7,0 & 8,6 & 13,0 & 7,0 & 7,0 & 9,9 \\
\hline $\begin{array}{l}\text { Зольность на сухую массу } A^{d}, \% \\
\text { Ash content on dry matter } A^{d}, \%\end{array}$ & 2,8 & 6,9 & 1,0 & 1,6 & 9,8 & 22,8 \\
\hline $\begin{array}{l}\text { Выход летучих веществ } V^{d a f}, \% \\
\text { Volatile substances } V^{\text {daf }}, \%\end{array}$ & 78,5 & 81,0 & 69,7 & 83,4 & 77,8 & 74,8 \\
\hline $\begin{array}{l}\text { Теплота сгорания на сухую беззольную массу } Q^{\text {daf }}, \text { МДж/кг } \\
\text { Calorific value for dry ashless mass } Q^{\text {daf }}, \mathrm{MJ} / \mathrm{kg}\end{array}$ & 19,6 & 19,1 & 21,0 & 19,8 & 14,8 & 20,1 \\
\hline $\begin{array}{l}\text { Низшая теплота сгорания } Q_{i}^{r}, \text { МДж/кг } \\
\text { Net calorific value } Q_{i}^{r}, \mathrm{MJ} / \mathrm{kg}\end{array}$ & 17,7 & 16,6 & 18,1 & 18,1 & 17,9 & 11,8 \\
\hline $\begin{array}{l}\text { Элементный состав на сухую беззольную массу, \% } \\
\text { Elemental composition for dry ashless mass, \% }\end{array}$ & & & & & & \\
\hline$C^{\text {daf }}$ & 50,20 & 49,14 & 51,81 & 52,5 & 47,87 & 52,06 \\
\hline$H^{\text {daf }}$ & 6,36 & 6,66 & 6,39 & 6,58 & 6,16 & 6,31 \\
\hline$N^{d a f}$ & 1,09 & 3,30 & 0,24 & 0,22 & 1,94 & 3,58 \\
\hline$S^{d a f}$ & следы & 0,07 & следы & следы & 0,25 & 0,20 \\
\hline$O^{d a f}$ & 42,35 & 40,83 & 41,56 & 40,70 & 43,78 & 37,85 \\
\hline$H / C$ атом. & 1,52 & 1,63 & 1,48 & 1,50 & 1,54 & 1,45 \\
\hline
\end{tabular}

Примечание: *-результаты элементного состава топлива приведень с учетом содержащегося в топливе диоксида углерода карбонатов $\left(\mathrm{CO}_{2}\right)^{d}=9,82 \%$ [58].

Note: * the results of fuel elemental composition are given taking into account carbon dioxide carbonates $\left(\mathrm{CO}_{2}\right)^{d}=9,82 \%$ [58].

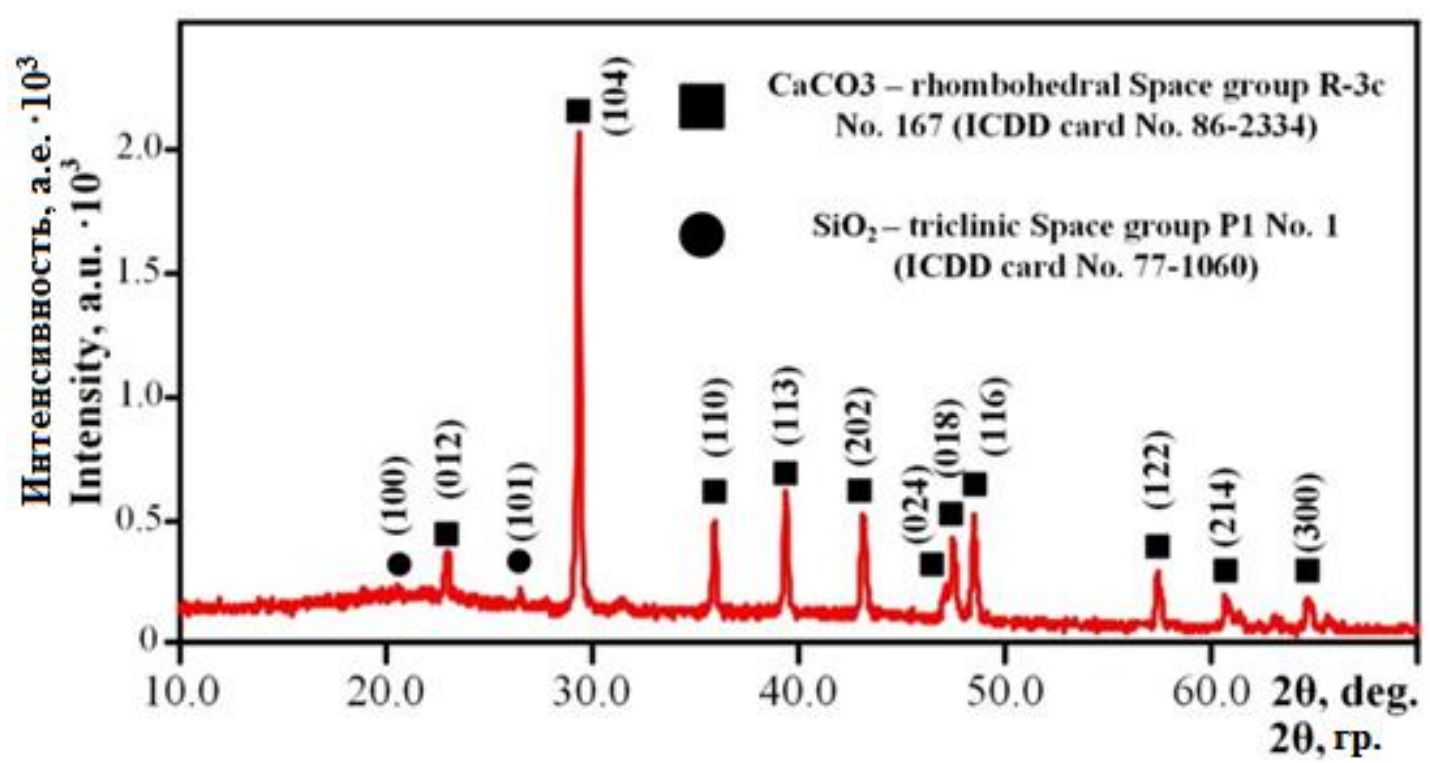

Pис. 1. Дифрактограмма рентгеноструктурного анализа суховского торфа

Fig. 1. X-ray diffraction pattern of Sukhovsky peat 


\section{Диффференциальный термический анализ}

На рис. 2 приведены результаты термогравиметрического анализа биомассы в интервале 313-1273 K. Видно, что в интервале температур 313-423 К происходит испарение влаги, содержащийся в образцах биомассы, после чего образцы нагреваются без изменения массы до температуры 463-488 К (в зависимости от вида биомассы). При этих температурах наблюдается начало первой стадии термического разложения биомассы, продолжающейся до 623-653 К и характеризующейся резким снижением массы образцов от 24 (торф) до $63 \%$ (опилки). Согласно литературным данным [59] этот температурный интервал разложения характерен для целлюлозы и гемицеллюлозы, содержащихся в большом количестве в биомас- се: содержание целлюлозы в биомассе может достигать 56 \%, гемицеллюлозы - $35 \%$ [60-65].

В интервале температур от 623-653 до 873 K наблюдается вторая (заключительная) стадия разложения биомассы, характеризующаяся значительно меньшим по сравнению с первой стадией изменением массы - от 9 до 14 \%. Дальнейшее увеличение температуры приводит лишь к несущественному изменению массы образцов. Исключение составляет суховской торф: в интервале температуры 923-1123 К его масса уменьшается ещё на $18 \%$. Однако это изменение, по-видимому, обусловлено разложением карбоната кальция $\mathrm{CaCO}_{3}$ на $\mathrm{CaO}$ и $\mathrm{CO}_{2}$, что подтверждается данными по изучению его термического разложения [66]. a/a)

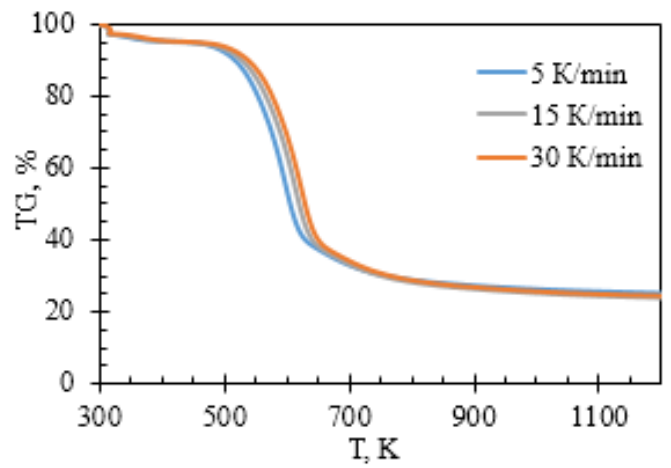

$\mathrm{B} / \mathrm{C})$

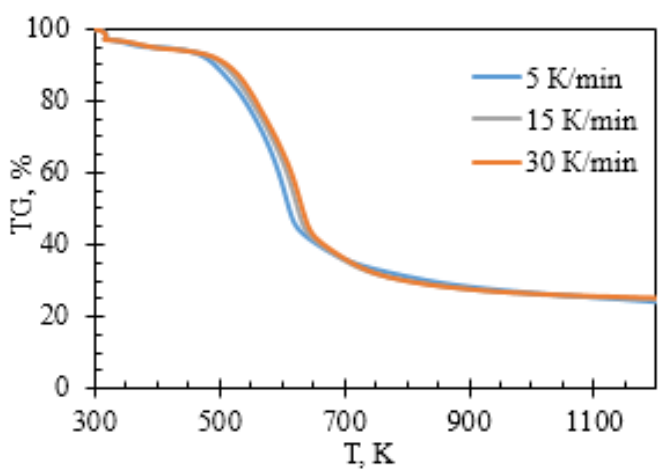

д/e)

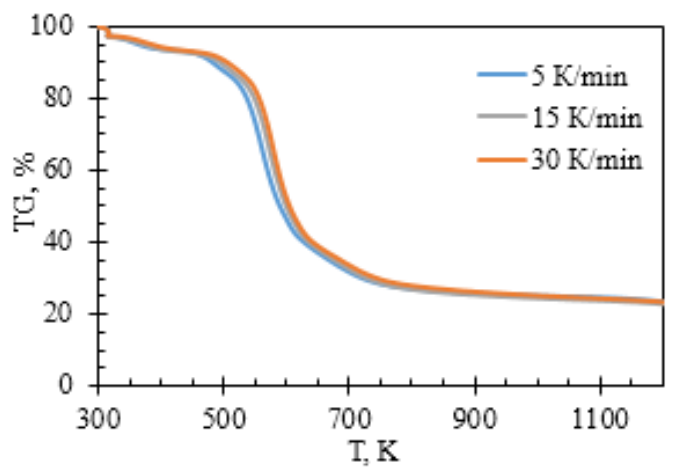

$6 / b)$

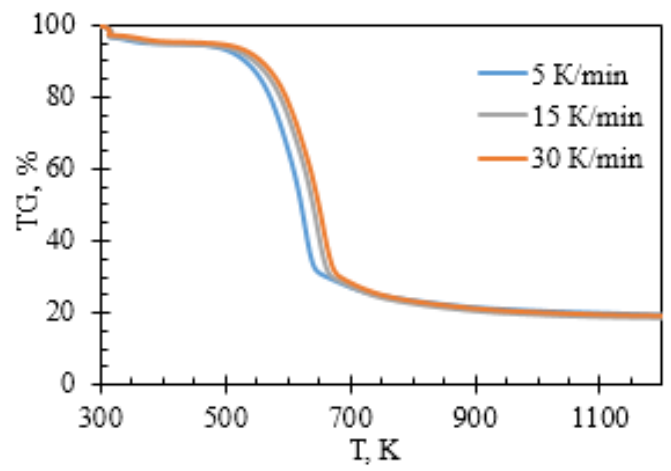

$\Gamma / d)$

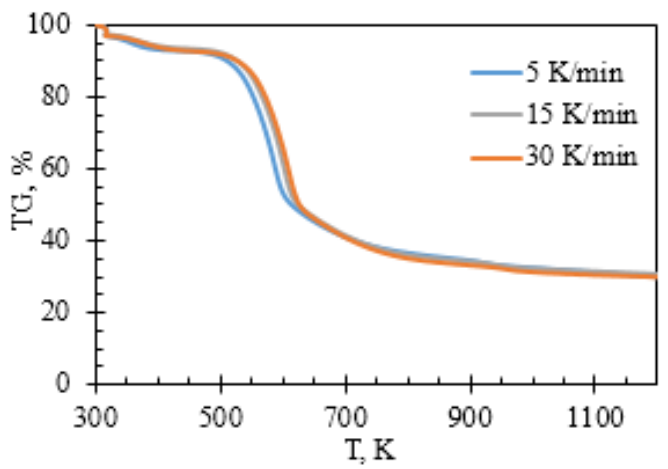

e/f)

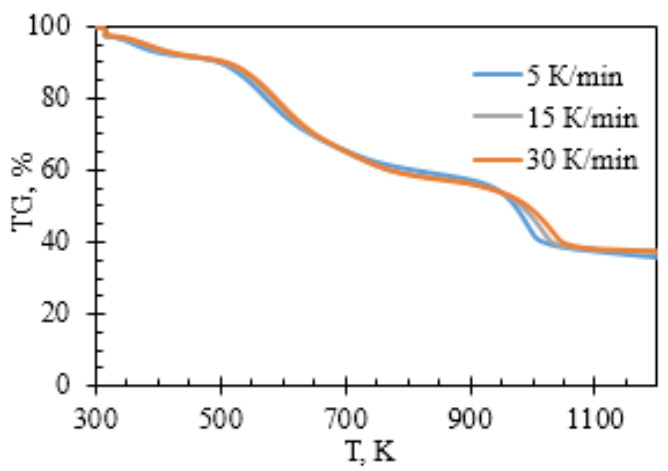

Рис. 2. Кривые термогравиметрического анализа (TG - thermogravimetric analysis) биомассы при скорости нагрева 5-30 K/мин: а) солома; б) опилки; в) скорлупа; г) ОЖКС; д) отруби; е) торф

Fig. 2. Thermogravimetric analysis (TG) curves of biomass at a speed of 5-30 K/min: a) straw; b) sawdust; c) shell; d) waste from cattle; e) bran; f) peat 
Стоит отметить, что увеличение скорости нагрева от 5 до $30 \mathrm{~K}$ в минуту не оказывает существенного воздействия на результаты ТГ, однако влияет на скорость изменения массы (дифференциальный термогравиметрический анализ - ДТГ) в зависимости от температуры нагрева (рис. 3). Видно, что скорость изменения массы прямо пропорциональна скорости

a/a)

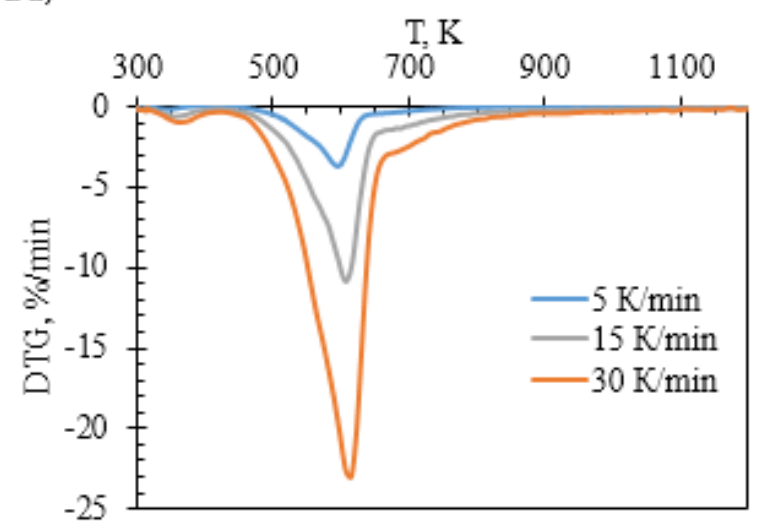

$\mathrm{B} / \mathrm{C})$

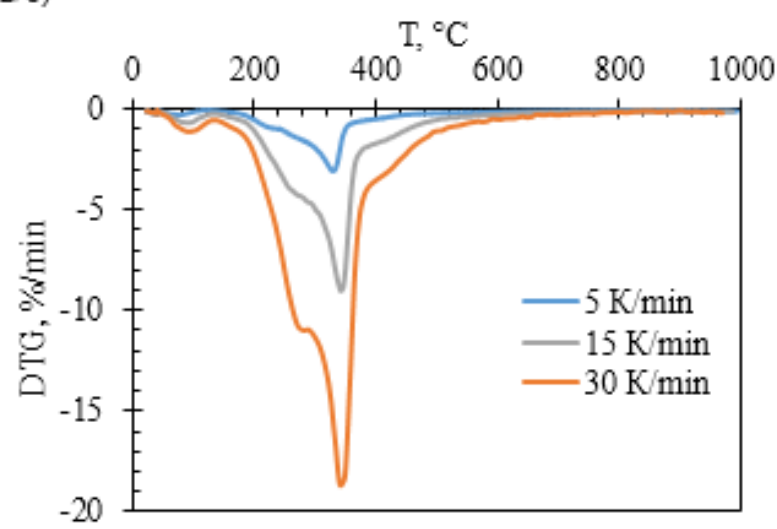

д/e)

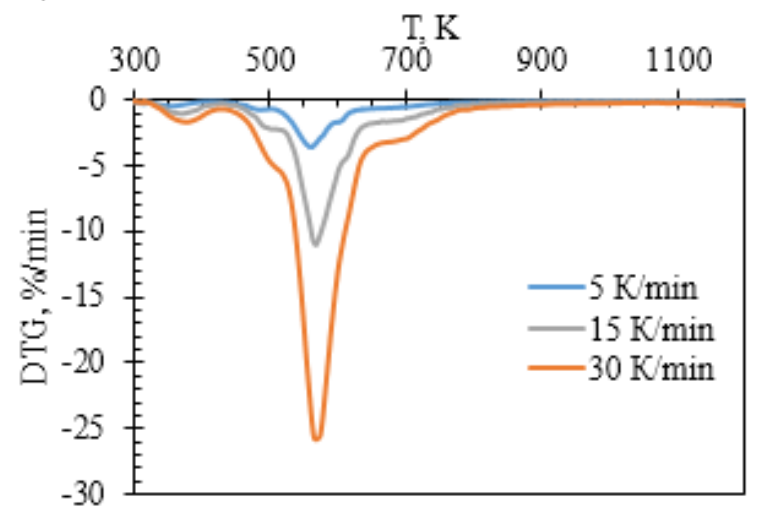

нагрева: увеличение скорости нагрева с 5 до 30 K/мин. привело к росту ДТГ в 6 раз.

Как видно из рис. 3, максимальная скорость разложения для соломы наблюдается при $613 \mathrm{~K}$, для опилок - при $643 \mathrm{~K}$, для скорлупы - при $613 \mathrm{~K}$, для ОЖКС - при 598 К, для отрубей - при 573 К, для торфа - при $583 \mathrm{~K}$.

$6 / b)$

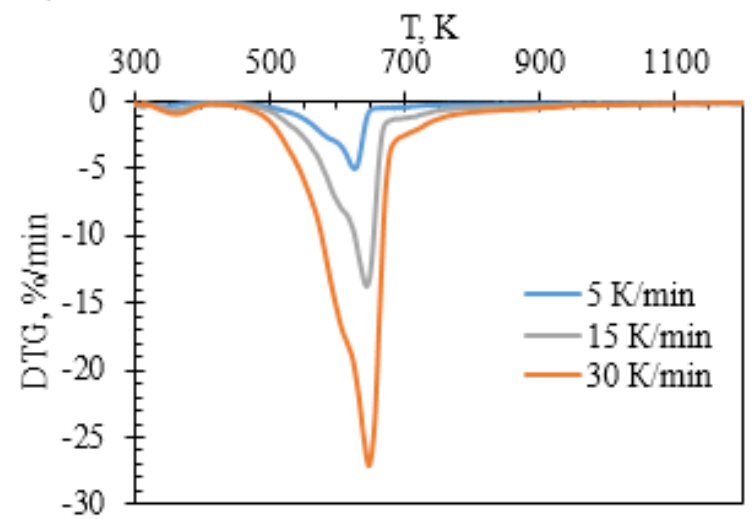

$\Gamma / d)$

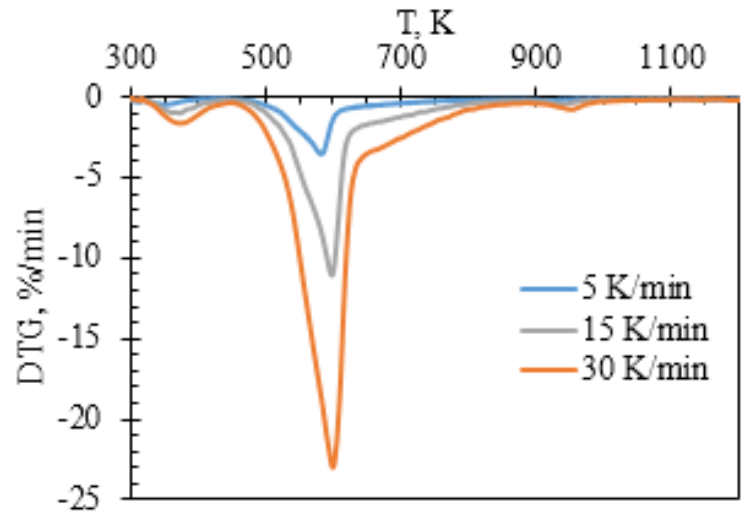

e/f)

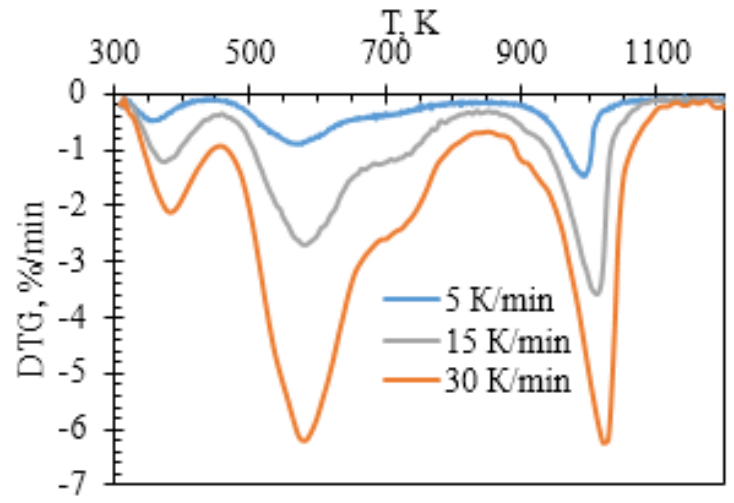

Рис. 3. Кривые ДТГ биомассы при скорости нагрева 5-30 К/мин: а) солома; б) опилки; в) скорлупа; г) ОЖКС; д) отруби; е) торф

Fig. 3. Differential thermogravimetric analysis (DTG) curves of biomass at heating rate of $5-30 \mathrm{~K} / \mathrm{min}$ : a) straw; b) sawdust; c) shell; d) waste from cattle; e) bran; f) peat

Результаты ДСК (рис. 4) показывают, что разложение всех исследованных проб в диапазоне температур от 463-488 до 873 К сопровождается преимущественно экзотермическими реакциями, связанными с разложением основных компонентов биомассы (гемицеллюлозы, целлюлозы, лигнина и др.). Это подтверждается ранее проведенными исследованиями различных авторов [67-69]. 
a/a)

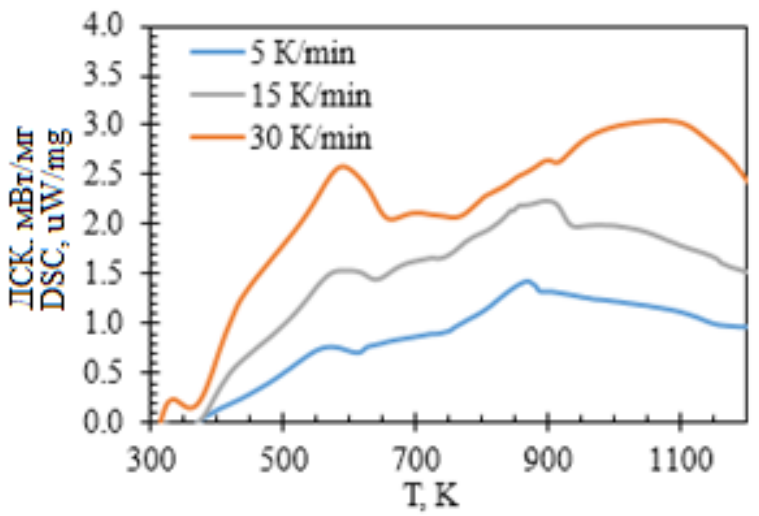

$\mathrm{B} / \mathrm{C})$

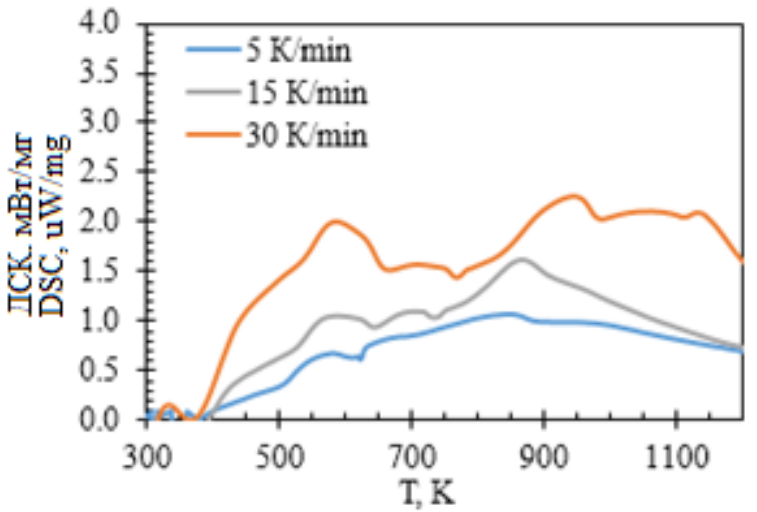

д/e)

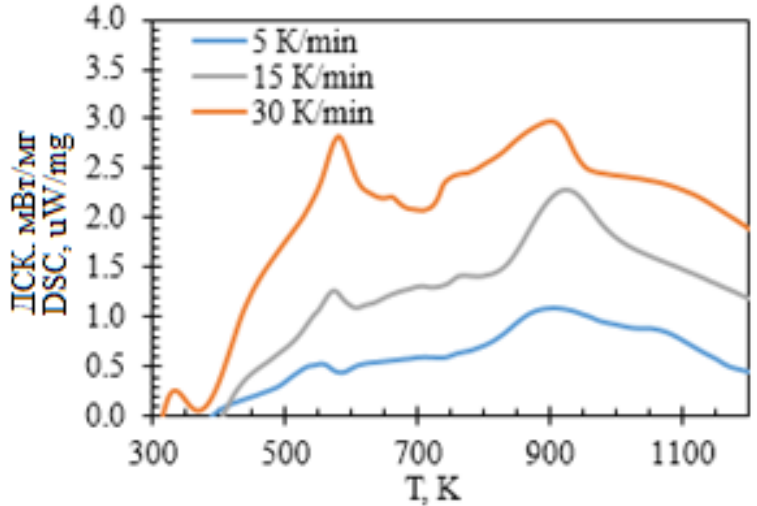

$6 / b)$

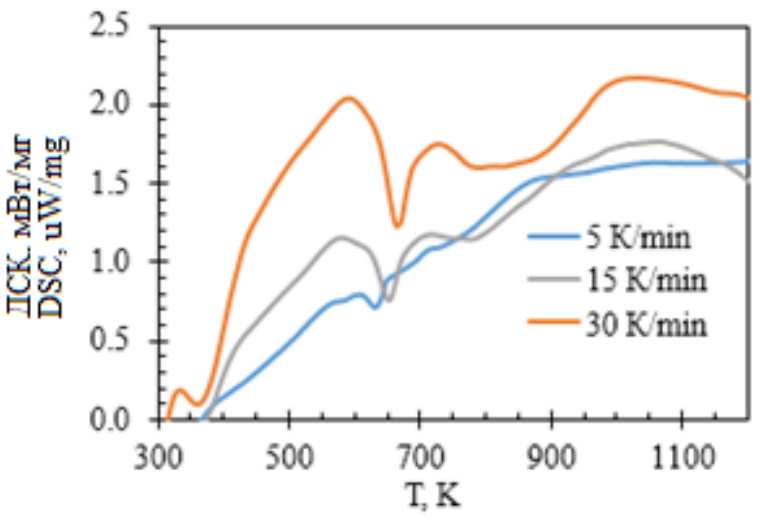

r/d)

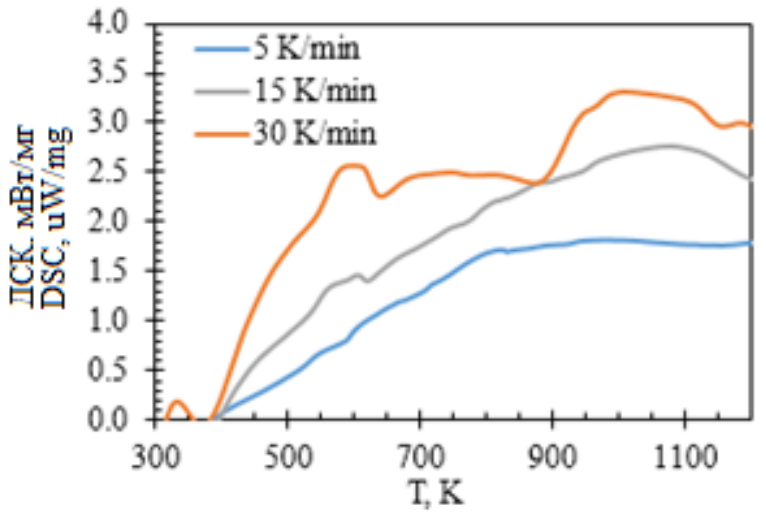

e/f)

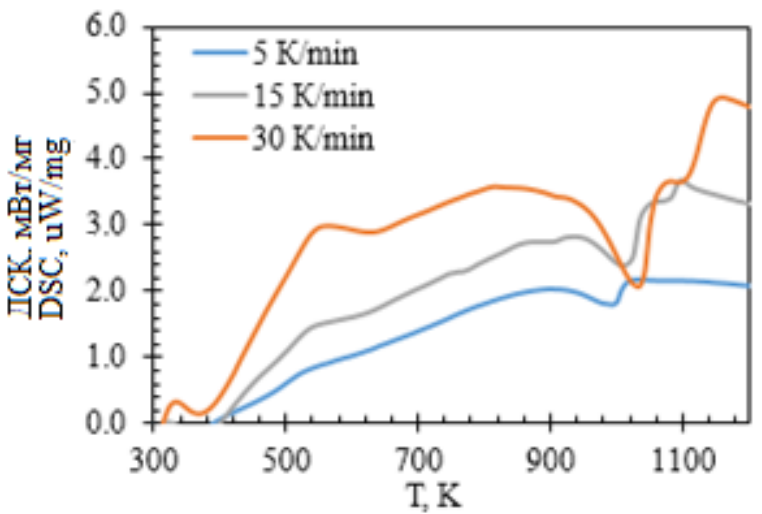

Pис. 4. Кривые ДСК биомассы при скорости нагрева 5-30 K/мин: а) солома; б) опилки; в) скорлупа; г) ОЖКС; д) отруби; е) торф

Fig. 4. Differential scanning calorimetry (DSC) curves of biomass at a heating rate of 5-30 K/min: a) straw; b) sawdust; c) shell; d) waste from cattle; e) bran; f) peat

\section{Кинетика термического разложения}

По результатам ТГ для трех скоростей нагрева (рис. 2) согласно (3) построены графики зависимости $\left(\ln \left(\left(-1 / w_{0}\right)(d w / d t)\right)\right)$ от $(1 / T)$ (рис. 5). Значения степени конверсии $\left(w / w_{0}\right)$ выбраны для каждого вида топлива индивидуально в зависимости от результатов ТГ. Шаг изменения $\left(w / w_{0}\right)$ принят равным 0,05 .

Значения $E_{a}$ и $\left(\ln \left[\left(A f\left(w / w_{0}\right)\right]\right)\right.$ для каждой пробы биомассы приведены на рис. 6 для всех рассматриваемых диапазонов степени конверсии $\left(w / w_{0}\right)$. Полученные зависимости $E_{a}$ и $\left(\ln \left[\left(A f\left(w / w_{0}\right)\right]\right)\right.$ применитель- но к каждому конкретному образцу имеют идентичный вид, что, согласно [39], свидетельствует о корректности выполненного расчета. Средние значения энергии активации для рассмотренных интервалов $\left(w / w_{0}\right)$ составляют: для соломы $\left(E a_{\mathrm{cp}}^{\mathrm{co}}\right)$ 21,4 кДж моль ${ }^{-1}$; для опилок $\left(E a_{\mathrm{cp}}^{\text {оп }}\right)-20,7$ кДж моль ${ }^{-1}$; для скорлупы $\left(E a_{\text {cр }}^{\text {ск }}\right)-23,8$ кДж моль ${ }^{-1}$; для ОЖКС $\left(E a_{\text {cp }}^{\text {н }}\right)-23,1$ кДж моль ${ }^{-1}$; для отрубей $\left(E a_{\text {cp }}^{\text {oт }}\right)-$ 31,5 кДж моль ${ }^{-1}$; для торфа $\left(E a_{\mathrm{cp}}^{\mathrm{T}}\right)-24,0$ кДж моль ${ }^{-1}$.

Из рис. 6 можно заключить, что, несмотря на близкий состав исследуемых проб биомассы (табл. 1), их 
термическое разложение протекает по различному механизму химических реакций (зависимость $\left(E_{a}\right)$ от $\left(w / w_{0}\right)$ имеет разный профиль для каждого вида биомассы). Можно отметить, что наибольшее значение $\left(E_{a}\right)=73$ кДж моль ${ }^{-1}$ наблюдается для отрубей при $\left(w / w_{0}\right)=0,46$, что соответствует $630 \mathrm{~K}$ - температура разложения целлюлозы [59]; второй пик - при температуре разложения гемицеллюлозы (555 К). При близких температурах (600-613 К) проявляется пик значений $\left(E_{a}\right)$ у соломы, опилок и ОЖКС. Можно прийти к выводу, что наибольшие значения энергии активации при пиролизе биомассы характерны для температур, при которых происходит разложение основных её компонентов (гемицеллюлозы, целлюлозы и лигнина).

При сравнении полученных значений с данными других авторов, производивших расчет энергии акти-

a/a)

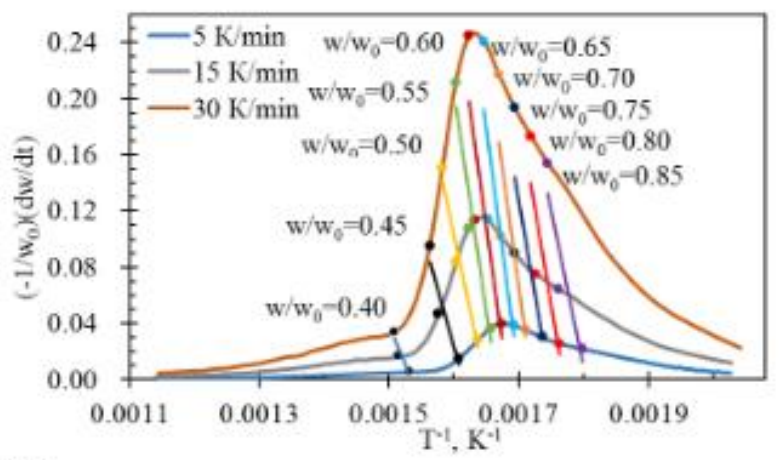

$\mathrm{B} / \mathrm{C}$ )

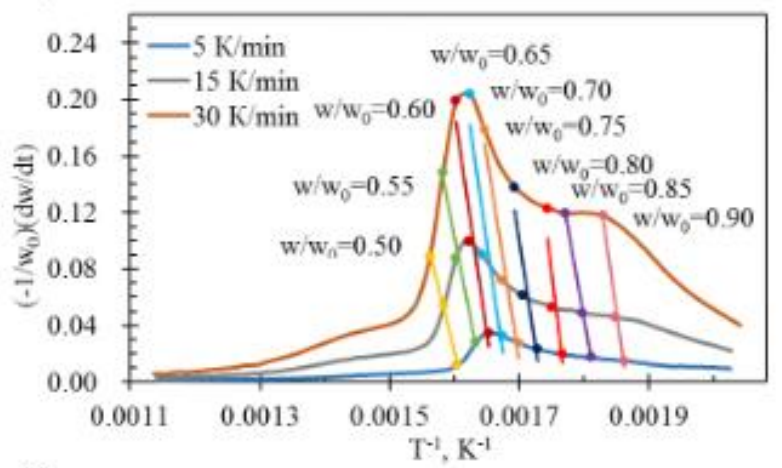

д/e)

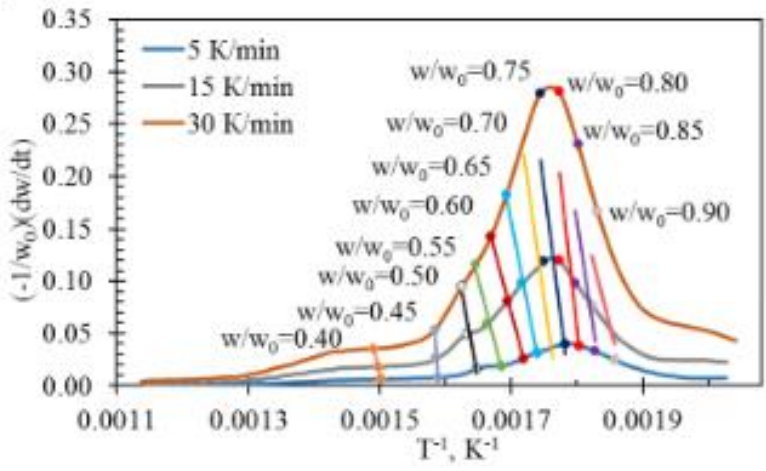

вации различными методами [42-45], можно отметить, что $\left(E_{a}\right)$ термического разложения биомассы Томской области имеет меньшие значения.

Согласно методике [39] на основе полученных результатов определен предэкспоненциальный множитель $A$ и порядок реакции $n$, значения которых представлены в табл. 2. Видно, что полученное значение $\mathrm{n}$ для реакций, происходящих при пиролизе биомассы, имеет более низкое значение, чем этот же параметр для реакций термического разложения отдельных её компонентов (целлюлозы, гемицеллюлозы и лигнина) [42]. Это можно объяснить более сложным механизмом разложения, обусловленным бо́льшим количеством одновременно протекающих реакций, оказывающих влияние друг на друга.

$6 / \mathrm{b})$

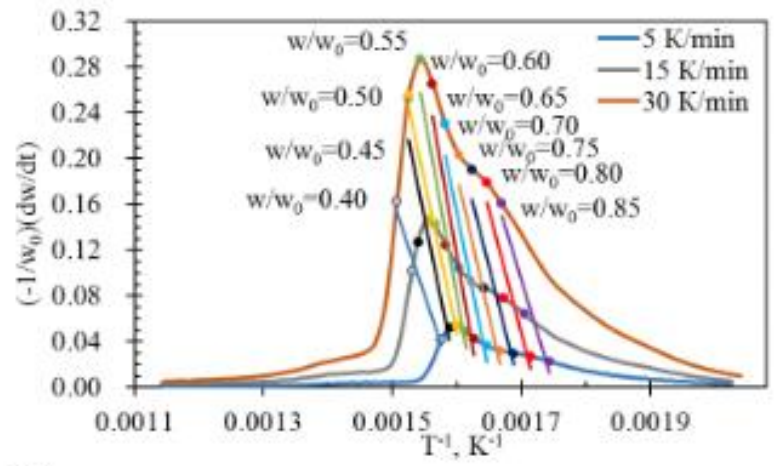

$\Gamma / d)$

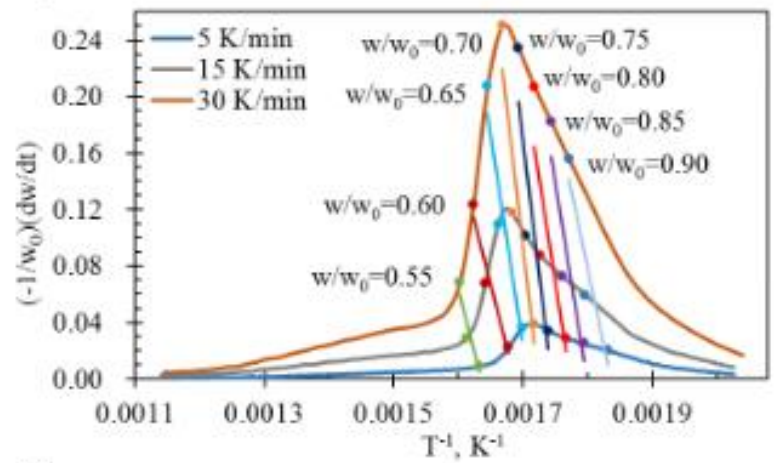

e/f)

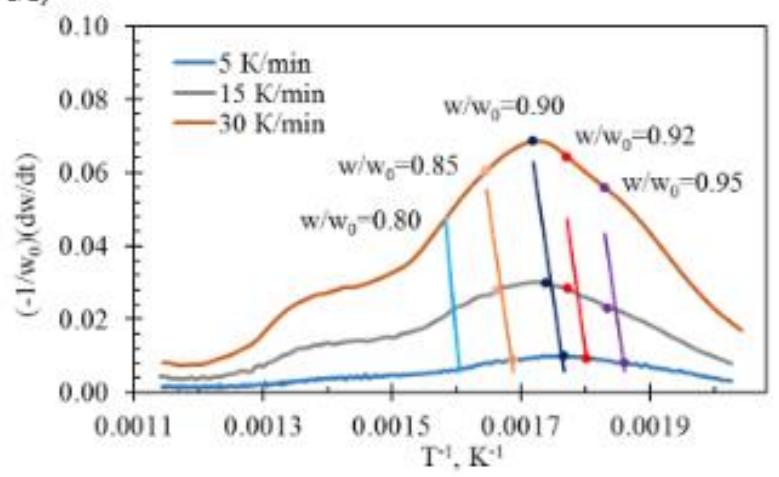

Рис. 5. Кривые зависимости $\left(\ln \left(\left(-1 / w_{0}\right)(d w / d t)\right)\right)$ от $(1 / T)$ для различных видов биомассы: а) солома; б) опилки; в) скорлупа; г) ОЖКС; д) отруби; е) торф

Fig. 5. Curves of dependence of $\left(\ln \left(\left(-1 / w_{0}\right)(d w / d t)\right)\right)$ on $(1 / T)$ for various types of biomass: a) straw; b) sawdust; c) shell; d) waste from cattle; e) bran; f) peat 
a/a)

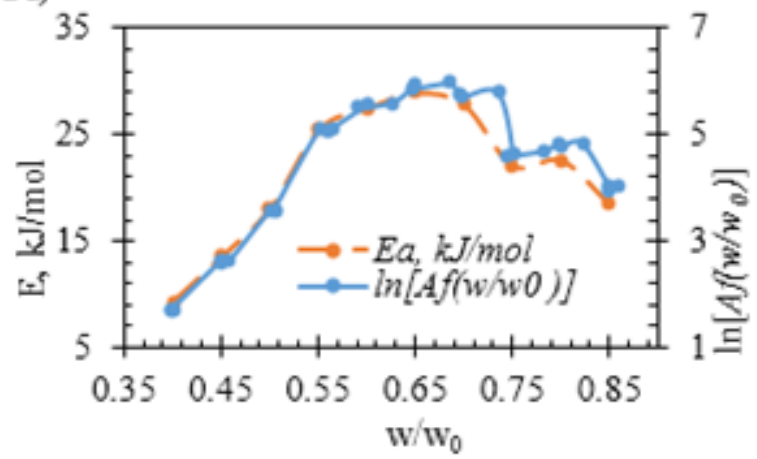

$\mathrm{B} / \mathrm{c})$

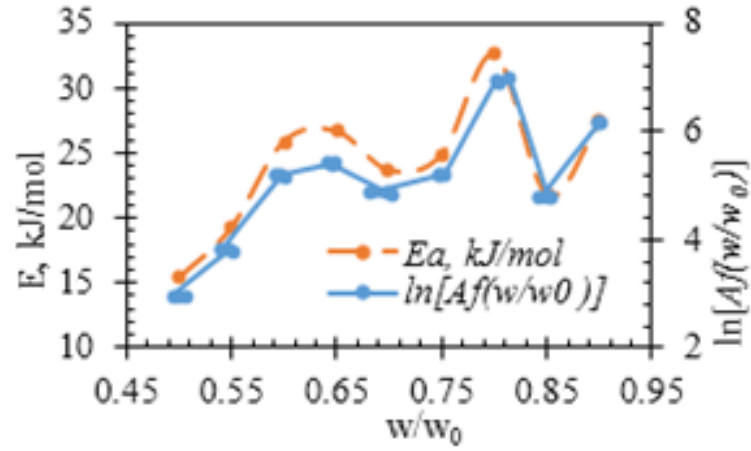

I/e)

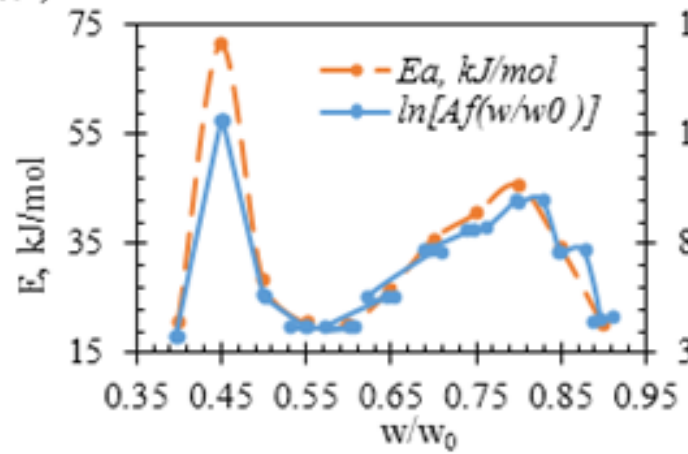

$6 / b)$

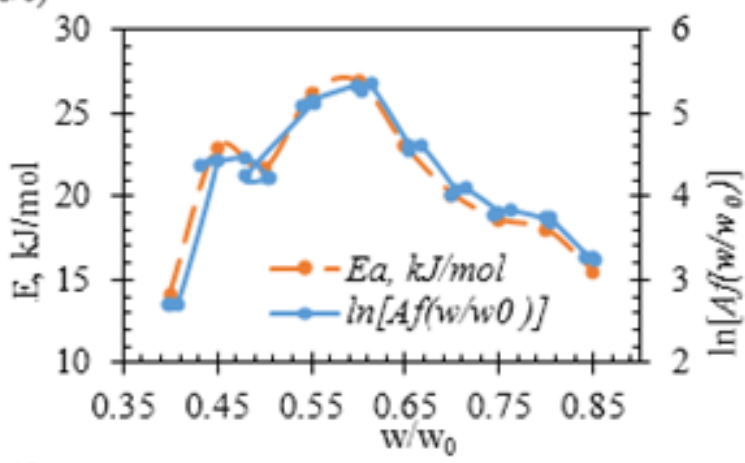

$\mathrm{r} / \mathrm{d})$

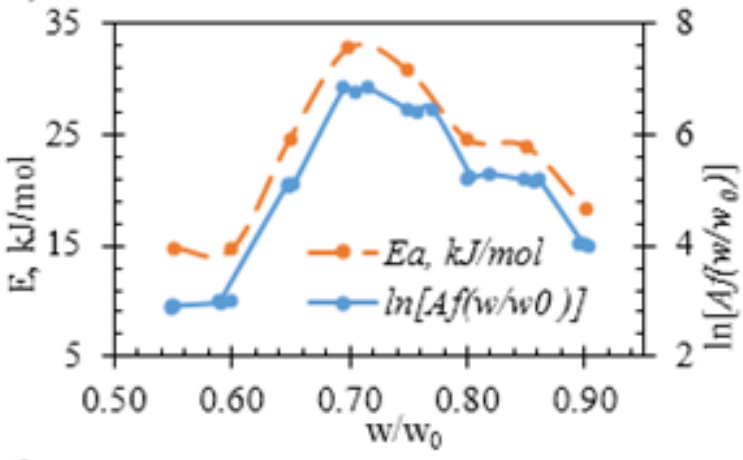

e/f)

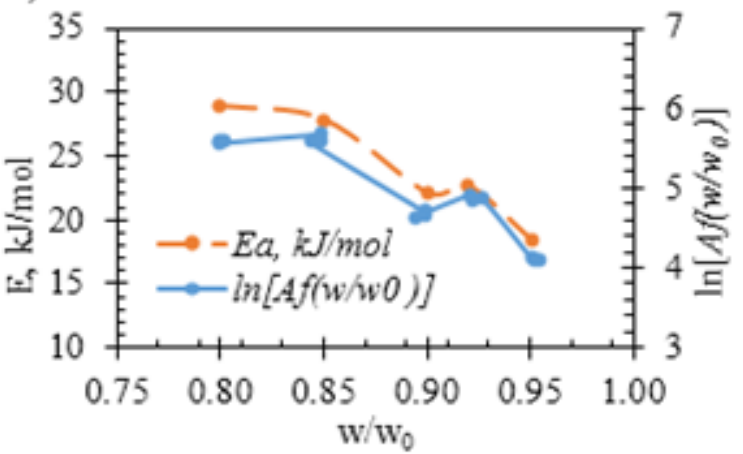

Pис. 6. Зависимость энергии активачии $E_{a}$ и $\ln \left[\left(A f\left(w / w_{0}\right)\right]\right.$ от степени конверсии биомассы в прочессе пиролиза: а) солома; б) опилки; в) скорлупа; г) ОЖКС; д) отруби; е) торф

Fig. 6. Dependence of the activation energy $E_{a}$ and $\ln \left[\left(A f\left(w / w_{0}\right)\right]\right.$ on biomass conversion degree during pyrolysis: a) straw; b) sawdust; c) shell; d) waste from cattle; e) bran; f) peat

Таблица 2. Значения предэкспонециального множителя и порядка реакиии при пиролизе фракиџй

Table 2. Values of the pre-exponential factor and reaction order during fraction pyrolysis

\begin{tabular}{|l|c|c|c|c|c|c|}
\hline \multirow{2}{*}{\multicolumn{1}{|c|}{ Показатель/Indicator }} & \multicolumn{9}{c|}{ Биомасса/Вiomass } \\
\cline { 2 - 7 } & $\begin{array}{c}\text { солома } \\
\text { straw }\end{array}$ & $\begin{array}{c}\text { отруби } \\
\text { bran }\end{array}$ & $\begin{array}{c}\text { скорлупа } \\
\text { shell }\end{array}$ & $\begin{array}{c}\text { опилки } \\
\text { sawdust }\end{array}$ & $\begin{array}{c}\text { ОЖКС } \\
\text { waste from cattle }\end{array}$ & $\begin{array}{c}\text { торф } \\
\text { peat }\end{array}$ \\
\hline $\begin{array}{l}\text { Предэкспоненциальный множитель } A, \mathrm{q}^{-1} \\
\text { Pre-ехропеntial factor } A, \mathrm{~h}^{-1}\end{array}$ & 123,95 & 82,42 & 277,44 & 208,62 & 2377,01 & 664,88 \\
\hline $\begin{array}{l}\text { Порядок реакции } n \\
\text { Reaction order } n\end{array}$ & 0,39 & 0,28 & 0,62 & 0,47 & 0,80 & 1,05 \\
\hline
\end{tabular}

\section{Заключение}

Дифференциальный термический анализ биомассы показал, что ее термическое разложение в интервале 313-1273 К происходит в две стадии: первая стадия протекает в интервале температур от 463-488 до 623-653 К и характеризуется резким снижением массы образцов от 24 (торф) до 63 \% (опилки); вторая стадия наблюдается от 623-653 до 873 K со значительно меньшим изменением массы образцов (от 9 до 14 \%). При дальнейшем увеличении температуры ор- ганическая часть образцов практически не претерпевала преобразования, изменения происходили только в минеральной части торфа при температуре 923-1123 К и были связаны с разложением карбоната кальция. Отмечено, что увеличение скорости нагрева от 5 до 30 К в минуту не оказывает существенного воздействия на результаты ТГ, однако влияет на скорость изменения массы (ДТГ) в зависимости от температуры нагрева: увеличение скорости нагрева с 5 до 30 К/мин привело к росту ДТГ в 6 раз. 
На основе данных ТГ методом Фридмана произведен расчет кинетики термического разложения биомассы. Определены зависимости энергии активации $\left(E_{a}\right)$ от степени конверсии биомассы $\left(w / w_{0}\right)$, по которым рассчитаны её средние значения: для соломы $\left(E a_{\mathrm{cp}}^{\mathrm{co}}\right)-21,4$ кДж моль ${ }^{-1} ;$ для опилок $\left(E a_{\mathrm{cp}}^{\text {oп }}\right)-$ 20,7 кДж моль ${ }^{-1} ;$ для скорлупы $\left(E a_{\text {ср }}^{\text {ск }}\right)-$ 24,2 кДж моль ${ }^{-1}$; для ОЖКС $\left(E a_{c p}^{\mathrm{H}}\right)-23,1$ кДж моль ${ }^{-1}$; для отрубей $\left(E a_{\text {cp }}^{\text {oт }}\right)-33,1$ кДж моль ${ }^{-1}$; для торфа $\left(E a_{\mathrm{cp}}^{\mathrm{T}}\right)-24,0$ кДж моль ${ }^{-1}$. Отмечено, что наибольшие значения энергии активации при пиролизе биомассы

\section{СПИСОК ЛИТЕРАТУРЬ}

1. World energy outlook special report 2016: energy and air pollution. - Washington: International Energy Agency Publ., 2016. - 9 p.

2. Drivers of stagnating global carbon intensity of electricity and the way forward / T. Goh, B.W. Ang, B. Su, H. Wang // Energy Policy. -2018 . - V. 113. - P. 149-156.

3. The contribution of outdoor air pollution sources to premature mortality on a global scale / J. Lelieveld, J.S. Evans, M. Fnais, D. Giannadaki, A. Pozzer // Nature - 2015. - V. 525 - P. 367-371.

4. Cohen J.E. Human population: the next half century // Science. 2003. - V. 302 (5648). - P. 1172-1175.

5. Lutz W., Sanderson W., Scherbov S. The coming acceleration of global population ageing // Nature. - 2008. - V. 451 (7179). P. 716-719.

6. Bongaarts J. Human population growth and the demographic transition // Philos Trans R SocLond B Biol Sci. - 2009. - V. 364. P. 2985-2990.

7. Poumanyvong P., Kaneko S. Does urbanization lead to less energy use and lower $\mathrm{CO}_{2}$ emissions? A cross-country analysis // Ecological Economics. - 2010. - V. 70. - P. 434-444.

8. Lund H., Mathiesen B.V. Energy system analysis of $100 \%$ renewable energy systems - the case of Denmark in years 2030 and 2050 // Energy. - 2009. - V. 34 (5). - P. 524-531.

9. Assessment of China's renewable energy contribution during the $12^{\text {th }}$ Five Year Plan / L. Hong, N. Zhou, D. Fridley, C. Raczkowski // Energy Policy. - 2013. - V. 62. - P. 1533-1543.

10. Kabak M., Dagdeviren M. Prioritization of renewable energy sources for Turkey by using a hybrid MCDM methodology // Energy Conversion and Management. - 2014. - V. 79. - P. 25-33.

11. Zero carbon energy system of South East Europe in 2050 D.F. Dominkovic, I. Bacekovic, B. Cosic, G. Krajacic, T. Puksec, N. Duic, N. Markovska // Applied Energy. - 2016. - V. 184. P. 1517-1528.

12. Ellabban O., Abu-Rub H., Blaabjerg F. Renewable energy re sources: current status, future prospects and their enabling technology // Renewable and Sustainable Energy Reviews. - 2014. V. 39. - P. 748-764.

13. Key world energy statistics. - Paris: International Energy Agency, 2018. $-51 \mathrm{p}$.

14. The first step towards a $100 \%$ renewable energy-system for Ireland / D. Connolly, H. Lund, B. Mathiesen, M. Leahy // Applied Energy. - 2011. - V. 88 (2). - P. 502-507.

15. How to achieve a $100 \%$ RES electricity supply for Portugal? G. Krajacic, N. Duic, M.D. Carvalho, I. Graca // Applied Energy. 2011. - V. 88 (2). - P. 508-517.

16. Deng Y.Y., Blok K., Leun K. Transition to a fully sustainable global energy system // Energy Strategy Reviews. - 2012. V. 1. - P. 109-121.

17. Elliston B., MacGill I., Diesendorf M. Comparing least cost sce narios for $100 \%$ renewable electricity with low emission fossil fuel scenarios in the Australian National Electricity Market // Renewable Energy. - 2014. - V. 66. - P. 196-204.

18. The reference forecast of the German energy transition - an outlook on electricity markets / A. Knaut, C. Tode, D. Lindenberger, R. Malischek, S. Paulus, J. Wagner // Energy Policy. - 2016. V. 92. - P. 477-491.

19. Goldemberg J., Coelho J.T., Suani I. Traditional biomass vs. modern biomass // Energy Policy. - 2004. - V. 32 (6). - P. 711-714. характерны для температур, при которых происходит разложение основных её компонентов (гемицеллюлозы, целлюлозы и лигнина).

Работа выполнена при поддержке Российского научного фонда (проект № 19-79-00085). Исследование теплотехнических характеристик и минеральной части биомассы проведено при поддержке Российского фонда фундаментальных исследований (проект № 19-38-90148). Аналитические измерения реализованы на приборной базе ТПУ в рамках государственного задания «Наука» (проект № FSWW-2020-0022).

20. Edenhofer O. The IPCC special report on renewable energy sources and climate change mitigation. - New York: Cambridge University Press, Intergovernmental Panel on Climate Change, 2011. - $1076 \mathrm{p}$.

21. McKendry P. Energy production from biomass (part 1): Overview of biomass // Bioresource Technology. - 2002. - V. 83 (1). - P. 37-46.

22. The amount of carbon released from peat and forest fires in Indonesia during 1997 / S.E. Page, F. Siegert, J.O. Rieley, H-D.V. Boehm, A. Jaya, S. Limin // Nature. - 2002. - V. 420. - P. 61-65.

23. Smoke emissions from biomass burning in a Mediterranean shrubland / C.A. Alves, C. Gonçalves, C.A. Pio, F. Mirante, A. Caseiro, L. Tarelho, M.C. Freitas, D.X. Viegas // Atmospheric Environment. - 2010. - V. 44 (25). - P. 3024-3033.

24. Interactions between climate, land use and vegetation fire occurrences in El Salvador / D. Armenteras, C. Gibbes, C.A. Vivacqua, J.S. Espinosa, W. Duleba, F. Goncalves, C. Castro // Atmosphere. - 2016. - V. 7 (2). - P. 26-41.

25. Global fire emissions and the contribution of deforestation, savanna, forest, agricultural, and peat fires (1997-2009) / G.R. van Der Werf, J.T. Randerson, L. Giglio, G.J. Collatz, M. Mu, P.S. Kasibhatla, D.C. Morton, R.S. Defries, Y. Jin, T.T. van Leeuwen // Atmos. Chem. Phys. - 2010. - V. 10. - P. 11707-11735.

26. Vassilev S.V., Vassileva C.G., Vassilev V.S. Advantages and disadvantages of composition and properties of biomass in comparison with coal: an overview // Fuel. - 2015. - V. 158. - P. 330-350.

27. Проблемы транспортировки угольных концентратов в период отрицательных температур окружающей среды / А.Д. Учитель, М.В. Кормер, В.П. Лялюк, И.А. Ляхова, Е.О. Шмельцер, Ю.И. Вититнев // Кокс и химия. - 2013. - № 5. - С. 13-19.

28. Performance of a pellet boiler fired with agricultural fuels / L. Carvalho, E. Wopienka, C. Pointner, J. Lundgren, V.K. Verma, W. Haslinger, C. Schmidl // Applied Energy. - 2013. - V. 104. P. 286-296.

29. The effect of co-combustion of waste from flour milling and highly mineralized peat on sintering of the ash residue / R. Tabakaev, K. Ibraeva, V. Kan, Y. Dubinin, M. Rudmin, N. Yazykov, A. Zavorin // Energy. - 2020. - V. 196. - P. 117-157.

30. Sutcu H. Pyrolysis of peat: product yield and characterization // Korean Journal of Chemical Engineering. - 2007. - V. 24. P. 736-741.

31. Jiang J., Ma X. Experimental research of microwave pyrolysis about paper mill sludge // Applied Thermal Engineering. - 2011. V. 31 (17-18). - P. 3897-3903.

32. Thermal enrichment of different types of biomass by lowtemperature pyrolysis / R. Tabakaev, I. Kanipa, A. Astafev, Y. Dubinin, N. Yazykov, A. Zavorin, V. Yakovlev // Fuel. 2019. - V. 245. - P. 29-38

33. Experimental study of catalytic pyrolysis of polyethylene and polypropylene over USY zeolite and separation to gasoline and diesel-like fuels / C. Kassargy, S. Awad, G. Burnens, K. Kahine, M. Tazerout // J. Anal. Appl. Pyrolys. - 2017. - V. 127. - P. 31-37.

34. Stepwise pyrolysis of mixed plastics and paper for separation of oxygenated and hydrocarbon condensates / N. Sophonrat, L. Sandstrom, I.N. Zaini, W. Yang // Appl. Energy. - 2018. - V. 229. P. 314-325.

35. Frank-Kamenetsky D.A. Diffusion and heat transfer in chemical kinetics. - New York: Plenum, 1969. - 574 p.

36. Helianthus tuberosus as a promising feedstock for bioenergy and chemicals appraised through pyrolysis, kinetics, and TG-FTIR-MS 
based study / M.A. Mehmood, M.S. Ahmad, Q. Liu, C-G. Liu, M.H. Tahir, A.A. Aloqbi, N.I. Tarbiah, H.M. Alsufiani, M. Gull // Energy Convers. Manage. - 2019. - V. 194. - P. 37-45.

37. Долганова И.О. Развитие подхода к моделированию процессов нефтепереработки на примере алкилирования бензолаэтиленом // Известия вузов. Прикладная химия и биотехнология. 2011. - № 1 (1). - C. 64-70.

38. Coats A.W., Redfern J.P. Kinetic parameters from thermogravimetric data // Nature. - 1964. - V. 201. - P. 68-69.

39. Friedman H.L. Kinetics of thermal degradation of char- forming plastics from thermogravimetry // Application to a phenolic plastic. -1964 . - V. 6C. - P. 183-195.

40. Model-free kinetic analysis of melamine-formaldehyde resin cure / A. Kandelbauer, G. Wuzella, A. Mahendran, I. Taudes, P. Widsten // Journal of Thermal Analysis and Calorimetry. - 2009. - V. 109 (3). - P. 1-10.

41. Lu C., Song W., Lin W. Kinetics of biomass catalytic pyrolysis // Biotechnology Advances - 2009. - V. 27 (5). - P. 583-587.

42. Гюльмалиев А.М., Султангузин И.А., Федюхин А.В. Математическое моделирование процесса пиролиза биомассы для производства синтез-газа и кокса // Химия твердого топлива. 2012. - № 3. - C. $25-29$.

43. Заварухин С.Г., Стрельцов И.А., Яковлев В.А. Исследование пиролиза быстрорастущей травянистой биомассы в изотермических условиях // Кинетика и катализ. - 2011. - Т. 52. № 4. - C. 510-516.

44. Kinetic modeling and optimization of parameters for biomass pyrolysis: A comparison of different lignocellulosic biomass / H. Mahmood, N. Ramzan, A. Shakeel, M. Moniruzzaman, T. Iqbal, M.A. Kazmi, M. Sulaiman // Energy Sources. P. A: Recovery, utilization and environmental effects. - 2019. - V. 41 (14). - P. 1690-1700.

45. Kinetic parameters for biomass under self-ignition conditions: lowtemperature oxidation and pyrolysis / L. Schwarzer, Z. Sárossy, P.A. Jensen, P. Glarborg, O. Karlström, J.K. Holm, K. DamJohansen // Energy and Fuels. - 2019. - V. 33 (9). - P. 8606-8619.

46. Alakangas E., Valtanen J., Levlin J.-E. CEN technical specification for solid biofuels-Fuel specification and classes // Biomass and Bioenergy. - 2006. - V. 30 (11). - P. 908-914.

47. Емешев В.Г., Паровинчак М.С. Без привозной энергетики // Нефтегазовая вертикаль. - 2005. - № 17. - С. 63-65.

48. Приложение к Постановлению Государственной Думы Томской области от 28.02.2008 № 1008 «Энергетическая стратегия Томской области на период до 2020 года». - Томск: Государственная Дума Томской области, 2008. - 89 с.

49. Торфяные ресурсы Томской области и их использование Л.И. Инишева, В.С. Архипов, С.Г. Маслов, Л.С. Михантьева. Новосибирск: Изд-во Сибирского отделения РАСХН, 1995. - 88 с.

50. ICTAC Kinetics Committee recommendations for collecting experimental thermal analysis data for kinetic computations / S. Vyazovkin, K. Chrissafis, M.L. Di Lorenzo, N. Koga, M. Pijolat, B. Roduit, N. Sbirrazzuoli, J.J. Suñol // ThermochimicaActa. 2014. - V. 590. - P. 1-23.

51. Bercic G. The universality of Friedman's isoconversional analysis results in a model-less prediction of thermodegradation profiles // ThermochimicaActa. - 2017. - V. 650. - P. 1-7.

52. Font R., Garrido M.A. Friedman and n-reaction order methods applied to pine needles and polyurethane thermal decompositions / ThermochimicaActa. - 2018. - V. 660. - P. 124-133.

53. Havilah P.R., Sharma P.K., Gopinath M. Combustion characteristics and kinetic parameter estimation of Lantana camera by thermogravimetric analysis // Biofuels. - 2019. - V. 10. - P. 365-372.
54. Izato Y.-I., Miyake A. Kinetic analysis of the thermal decomposition of liquid ammonium nitrate based on thermal analysis and detailed reaction simulations // Journal of Thermal Analysis and Calorimetry. -2018 . - V. 134. - P. 813-823.

55. Ash contents and ash-forming elements of biomass and their significance for solid biofuel combustion / S.V. Vassilev, C.G. Vassileva, Y. Song, W. Li, J. Feng // Fuel. - 2017. - V. 208. - P. 377-409.

56. Agro-pellets for domestic heating boilers: Standard laboratory and real life performance / V.K. Verma, S. Bram, F. Delattin, P. Laha, I. Vandendael, A. Hubin, J. De Ruyck // Applied Energy. 2012. - V. 90. - P. 17-23.

57. DIN plus pellets or EN plus pellets // AzeusBiopellet Machines. URL: https://www.biopelletmachine.com/biopellet-makingguidance/wood-pellet-certification-standard.html (дата обращения 25.02.2020).

58. Исследование характеристик и минерального состава торфа Томской области применительно к энергетическому использованию / К.Т. Ибраева, Ю.О. Манаев, Р.Б. Табакаев, Н.А. Языков, А.С. Заворин // Известия Томского политехнического университета. Инжиниринг георесурсов. - 2019. T. 330. - № 1. - C. 191-200.

59. Кузнецов Б.Н. Каталитическая химия растительной биомассы // Соровский образовательный журнал. - 1996. - № 12. - С. 47-55.

60. Mohan D., Pittman C.U. Jr., Steele P.H. Pyrolysis of wood/biomass for bio-oil: a critical review // Energy and Fuels. 2006. - V. 20. - P. 848-889.

61. Simoneit B.R.T. Biomass burning - a review of organic tracers for smoke from incomplete combustion // Applied Geochemistry. 2002. - V. 17. - P. 129-162.

62. Biomass yield and changes in chemical composition of sweet sorghum cultivars grown for biofuel / Y.L. Zhao, A. Dolat, Y. Steinberger, X. Wang, A. Osman, G.H. Xie // Field Crops Research. - 2009. - V. 111. - P. 55-64.

63. Сивенков А.Б. Влияние физико-химических характеристик древесины на ее пожарную опасность и эффективность огнезащиты: автореф. дис. ... д-ра техн. наук. - М., 2015. - 49 с.

64. Thermochemical biofuel production in hydrothermal media: a review of sub- and supercritical water technologies / A.A. Peterson, F. Vogel, R.P. Lachance, M. Froling, M.J. Antal Jr., J.W. Tester // Energy and Environmental Science. - 2008. - V. 1. - P. 32-65.

65. Gronli M.G., Varhegyi G., Di Blasi C. Thermogravimetric analysis and devolatilization kinetics of wood // Industrial and Engineering Chemistry Research. - 2002. - V. 41. - P. 4201-4208.

66. Капаев Г.И. Физико-химические основы процесса термического разложения солей угольной кислоты: автореф. дис. ... канд. хим. наук. - М., 2009. -20 c.

67. Exothermic events of nut shell and fruit stone pyrolysis C.D. Blasi, A. Galgano, C. Branca // ACS Sustainable Chemistry and Engineering. - 2019. - V. 7. - P. 9035-9049.

68. Autothermal pyrolysis of biomass due to intrinsic thermal decomposition effects / R.B. Tabakaev, A.V. Astafev, Y.V. Dubinin, N.A. Yazykov, A.S. Zavorin, V.A. Yakovlev // Journal of Thermal Analysis and Calorimetry. - 2018. - V. 134. - P. 1045-1057.

69. Chen X., Zhang H., Xiao R. Mobile autothermal pyrolysis system for local biomass conversion: process simulation and technoeconomic analysis // Energy and Fuels. - 2018. - V. 32. P. $4178-4188$

Поступила 18.11.2020 г.

\section{Информация об авторах}

Tабакаев P.Б., кандидат технических наук, научный сотрудник научно-образовательного центра И.Н. Бутакова Инженерной школы энергетики Национального исследовательского Томского политехнического университета. Алтынбаева Д.Б., студент научно-образовательного центра И.Н. Бутакова Инженерной школы энергетики Национального исследовательского Томского политехнического университета.

Ибраева К.T., аспирант, инженер-исследователь научно-образовательного центра И.Н. Бутакова Инженерной школы энергетики Национального исследовательского Томского политехнического университета.

Заворин A.C., доктор технических наук, профессор, заведующий кафедрой - руководитель научнообразовательного центра И.Н. Бутакова на правах кафедры Инженерной школы энергетики Национального исследовательского Томского политехнического университета. 
UDC 662.638

\title{
KINETIC CHARACTERISTICS OF BIOMASS PYROLYSIS
}

\author{
Roman B. Tabakaev 1 , \\ tabakaevRB@tpu.ru \\ Dariga B. Altynbaeva ${ }^{1}$, \\ dba10@tpu.ru \\ Kanipa T. Ibraeva ${ }^{1}$, \\ kti1@tpu.ru
}

\author{
Alexander S. Zavorin ${ }^{1}$, \\ zavorin@tpu.ru \\ ${ }^{1}$ National Research Tomsk Polytechnic University, \\ 30, Lenin avenue, Tomsk, 634050, Russia.
}

The relevance of the study is caused by the tendency to increase the share of renewable energy in the fuel and energy balance to reduce the harmful effects on the environment.

The main aim is to determine the kinetic parameters of biomass pyrolysis by G. Friedman's method based on the results of differential thermal analysis.

Objects: straw, grain processing waste (wheat bran), cedar nutshell, pine sawdust, lowland peat of the Sukhovskoe field, as well as livestock waste from cattle.

Methods. Thermotechnical characteristics of biomass samples are determined according to certified methods (GOST 11305-2013, GOST 11306-2013, GOST R 54186-2010, GOST R 56881-2016, GOST 32990-2014); values of the calorific value were determined using the ABK-1 calorimeter (RET, Russia) in accordance with GOST 147-2013 (ISO 1928-2009); elemental analysis of its organic part was carried out on a Vario Micro Cube (Elementar, Germany) device; the kinetic characteristics of low-temperature pyrolysis of biomass were determined by $\mathrm{G}$. Friedman's method based on differential thermal analysis carried out at temperature of 313-1273 K at a heating rate of 5, 15 and $30 \mathrm{~K} / \mathrm{min}$.

Results. Thermal decomposition of biomass in the range of 313-1273 K occurs in two stages: the first stage proceeds in the temperature range from 463-488 to 623-653 $\mathrm{K}$ and is characterized by a sharp decrease in the mass of samples from 24 (peat) to $63 \%$ (sawdust); the second stage is observed from 623-653 to $873 \mathrm{~K}$ with a significantly smaller change in the mass of the samples (from 9 to $14 \%$ ). With a further increase in temperature, the organic part of the samples practically did not undergo transformation, changes occurred only in the mineral part of peat at a temperature of 923-1123 $\mathrm{K}$ and were associated with the decomposition of calcium carbonate. The dependences of activation energy $\left(E_{a}\right)$ on the degree of biomass conversion $\left(w / w_{0}\right)$ were determined, according to which its average values were calculated: for straw $\left(E a_{\mathrm{cp}}^{s t}\right)-21,4 \mathrm{~kJ} \mathrm{~mol}^{-1}$; for sawdust $\left(E a_{\mathrm{cp}}^{s w}\right)-20,7 \mathrm{~kJ} \mathrm{~mol}^{-1}$; for shell $\left(E a_{\mathrm{cp}}^{s h}\right)-24,2 \mathrm{~kJ} \mathrm{~mol}^{-1}$; for livestock waste from cattle $\left(E a_{\mathrm{cp}}^{\mathrm{LW}}\right)-23,1 \mathrm{~kJ} \mathrm{~mol}^{-1}$; for bran $\left(E a_{\mathrm{cp}}^{\mathrm{br}}\right)-33,1 \mathrm{~kJ} \mathrm{~mol}^{-1}$; for peat $\left(E a_{\mathrm{cp}}^{p e}\right)-24,0 \mathrm{~kJ} \mathrm{~mol}^{-1}$. The values of the pre-exponential factor (A) for the studied biomass species are in the range of $82,42-2377,01 h^{-1}$.

\section{Key words:}

Biomass, thermal processing, differential thermal analysis, kinetics, Friedman's method.

This work was supported by the Russian Science Foundation (project no. 19-79-00085). The study of thermal characteristics and the mineral part of biomass was carried out with the support of the Russian Foundation for Basic Research (project no. 19-38-90148). Analytical measurements were implemented on the TPU instrument base within the framework of the state task «Science» (project no. FSWW-2020-0022).

\section{REFERENCES}

1. World energy outlook special report 2016: energy and air pollution. Washington, International Energy Agency, 2016. 9 p.

2. Goh T., Ang B.W., Su B., Wang H. Drivers of stagnating global carbon intensity of electricity and the way forward. Energy Policy, 2018, vol. 113, pp.149-156.

3. Lelieveld J., Evans J.S., Fnais M., Giannadaki D., Pozzer A. The contribution of outdoor air pollution sources to premature mortality on a global scale. Nature, 2015, vol. 525, pp. 367-371.

4. Cohen J.E. Human population: the next half century. Science, 2003, vol. 302 (5648), pp. 1172-1175.

5. Lutz W., Sanderson W., Scherbov S. The coming acceleration of global population ageing. Nature, 2008, vol. 451 (7179), pp. 716-719.

6. Bongaarts J. Human population growth and the demographic transition. Philosophical Transactions of the Royal Society B: Biological Sciences, 2009, vol. 364, pp. 2985-2990.
7. Poumanyvong P., Kaneko S. Does urbanization lead to less energy use and lower $\mathrm{CO}_{2}$ emissions? A cross-country analysis. Ecological Economics, 2010, vol. 70, pp. 434-444.

8. Lund H., Mathiesen B.V Energy system analysis of $100 \%$ renewable energy systems - the case of Denmark in years 2030 and 2050. Energy, 2009, vol. 34 (5), pp. 524-531.

9. Hong L., Zhou N., Fridley D., Raczkowski C. Assessment of China's renewable energy contribution during the $12^{\text {th }}$ Five Year Plan. Energy Policy, 2013, vol. 62, pp. 1533-1543.

10. Kabak M., Dagdeviren M. Prioritization of renewable energy sources for Turkey by using a hybrid MCDM methodology. Energy Conversion and Management, 2014, vol. 79, pp. 25-33.

11. Dominkovic D.F., Bacekovic I., Cosic B., Krajacic G., Puksec T., Duic N., Markovska N. Zero carbon energy system of South East Europe in 2050. Applied Energy, 2016, vol. 184, pp. 1517-1528.

12. Ellabban O., Abu-Rub H., Blaabjerg F. Renewable energy resources: current status, future prospects and their enabling tech- 
nology. Renewable and Sustainable Energy Reviews, 2014, vol. 39, pp. 748-764.

13. Key world energy statistics. Paris, International Energy Agency, 2018. $51 \mathrm{p}$.

14. Connolly D., Lund H., Mathiesen B., Leahy M. The first step towards a $100 \%$ renewable energy-system for Ireland. Applied Energy, 2011, vol. 88 (2), pp. 502-507.

15. Krajacic I., Duic G., Carvalho N., Graca M.D. How to achieve a $100 \%$ RES electricity supply for Portugal? Applied Energy, 2011, vol. 88 (2), pp. 508-517.

16. Deng Y.Y., Blok K., Leun K. Transition to a fully sustainable global energy system. Energy Strategy Reviews, 2012, vol. 1, pp. 109-121.

17. Elliston B., MacGill I., Diesendorf M. Comparing least cost scenarios for $100 \%$ renewable electricity with low emission fossil fuel scenarios in the Australian National Electricity Market. Renewable Energy, 2014, vol. 66, pp. 196-204.

18. Knaut A., Tode C., Lindenberger D., Malischek R., Paulus S., Wagner J. The reference forecast of the German energy transition - an outlook on electricity markets. Energy Policy, 2016, vol. 92, pp. 477-491.

19. Goldemberg J., CoelhoJ. T., Suani I. Traditional biomass vs. modern biomass. Energy Policy, 2004, vol. 32 (6), pp. 711-714.

20. Edenhofer O. The IPCC special report on renewable energy sources and climate change mitigation. New York, Cambridge University Press, Intergovernmental Panel on Climate Change, 2011. $1076 \mathrm{p}$

21. McKendry P. Energy production from biomass (part 1): Overview of biomass. Bioresource Technology, 2002, vol. 83 (1), pp. 37-46.

22. Page S.E., Siegert F., Rieley J.O., Boehm H-D.V., Jaya A., Limin S. The amount of carbon released from peat and forest fires in Indonesia during 1997. Nature, 2002, vol. 420, pp. 61-65.

23. Alves C.A., Gonçalves C., Pio C.A., Mirante F., Caseiro A., Tarelho L., Freitas M.C., Viegas D.X. Smoke emissions from biomass burning in a Mediterranean shrubland. Atmospheric Environment, 2010, vol. 44 (25), pp. 3024-3033.

24. Armenteras D., Gibbes C., Vivacqua C.A., Espinosa J.S., Duleba W., Goncalves F., Castro C. Interactions between climate, land use and vegetation fire occurrences in El Salvador. Atmosphere, 2016, vol. 7 (2), pp. 26-41.

25. Van Der Werf G.R., Randerson J.T., Giglio L., Collatz G.J., Mu M., Kasibhatla P.S., Morton D.C., Defries R.S., Jin Y., Van Leeuwen T.T. Global fire emissions and the contribution of deforestation, savanna, forest, agricultural, and peat fires (1997-2009). Atmospheric Chemistry and Physics, 2010, vol. 10, pp. 11707-11735.

26. Vassilev S.V., Vassileva C.G., Vassilev V.S. Advantages and disadvantages of composition and properties of biomass in comparison with coal: an overview. Fuel, 2015, vol. 158, pp. 330-350.

27. Uchitel A.D., Kormer M.V., Lyalyuk V.P., Lyakhova I.A., Shmeltser E.O., Vititnev Y.I. Transportation of coal concentrates at negative ambient temperatures. Coke and Chemistry, 2013, vol. 56, no. 5, pp. 13-19. In Rus.

28. Carvalho L., Wopienka E., Pointner C., Lundgren J., Verma V.K., Haslinger W., Schmidl C. Performance of a pellet boiler fired with agricultural fuels. Applied Energy, 2013, vol. 104, pp. 286-296.

29. Tabakaev R., Ibraeva K., Kan V., Dubinin Y., Rudmin M., Yazykov N., Zavorin A. The effect of co-combustion of waste from flour milling and highly mineralized peat on sintering of the ash residue. Energy, 2020, vol. 196, pp. 117-157.

30. Sutcu H. Pyrolysis of peat: product yield and characterization. Korean Journal of Chemical Engineering, 2007, vol. 24, pp. 736-741

31. Jiang J., Ma X. Experimental research of microwave pyrolysis about paper mill sludge. Applied Thermal Engineering, 2011, vol. 31 (17-18), pp. 3897-3903.

32. Tabakaev R., Kanipa I., Astafev A., Dubinin Y., Yazykov N., Zavorin A., Yakovlev V. Thermal enrichment of different types of biomass by low-temperature pyrolysis. Fuel, 2019, vol. 245, pp. 29-38.

33. Kassargy C., Awad S., Burnens G., Kahine K., Tazerout M. Experimental study of catalytic pyrolysis of polyethylene and polypropylene over USY zeolite and separation to gasoline and diesellike fuels. Journal of Analytical and Applied Pyrolysis, 2017, vol. 127 , pp. 31-37.

34. Sophonrat N., Sandstrom L., Zaini I.N., Yang W. Stepwise pyrolysis of mixed plastics and paper for separation of oxygenated and hydrocarbon condensates. Applied Energy, 2018, vol. 229, pp. 314-325.

35. Frank-Kamenetsky D.A. Diffusion and heat transfer in chemical kinetics. New York, Plenum Publ., 1969. 574 p.

36. Mehmood M.A., Ahmad M.S., Liu Q., Liu C-G., Tahir M.H., Aloqbi A.A., Tarbiah N.I., Alsufiani H.M., Gull M. Helianthus tuberosus as a promising feedstock for bioenergy and chemicals appraised through pyrolysis, kinetics, and TG-FTIR-MS based study. Energy Conversion Management, 2019, vol. 194, pp. 37-45.

37. Dolganova I.O. Razvitie podkhoda $\mathrm{k}$ modelirovaniyu protsessov nneftepererabotki na primere alkilirovaniya benzolaetilenom [Development of an approach to the modeling of oil refining processes by the example of benzene alkylation with ethylene]. Izvestiva vyzov. Prikladnaya khimiya i biotekhnologiya, 2011, no. 1 (1), pp. 64-70.

38. Coats A.W., Redfern J.P. Kinetic parameters from thermogravimetric data. Nature, 1964, vol. 201, pp. 68-69.

39. Friedman H.L. Kinetics of thermal degradation of char- forming plastics from thermogravimetry. Application to a phenolic plastic, 1964, vol. 6C, pp. 183-195.

40. Kandelbauer A., Wuzella G., Mahendran A., Taudes I., Widsten P. Model-free kinetic analysis of melamine-formaldehyde resin cure. Journal of Thermal Analysis and Calorimetry, 2009, vol. 109 (3), pp. 1-10.

41. Lu C., Song W., Lin W. Kinetics of biomass catalytic pyrolysis. Biotechnology Advances, 2009, vol. 27 (5), pp. 583-587.

42. Gulmaliev A.M., Sultanguzin I.A., Fedukhin A.V. Matematicheskoe modelirovanie protsessa piroliza biomassy dlya proizvodstva sintez-gaza i koksa [Mathematical modeling of the biomass pyrolysis process for the production of synthesis gas and coke]. Khimiya tverdogo topliva, 2012, no. 3, pp. 25-29.

43. Zavarukhin S.G., Streltsov I.A., Yakovlev V.A. Issledovanie piroliza bystrorastushchey travyanistoy biomassy $\mathrm{v}$ izotermicheskikh usloviykah [The study of pyrolysis of fast-growing grassy biomass in isothermal conditions]. Kinetika i kataliz, 2011, vol. 52, no. 4 , pp. $510-516$

44. Mahmood H., Ramzan N., Shakeel A., Moniruzzaman M., Iqbal T., Kazmi M.A., Sulaiman M. Kinetic modeling and optimization of parameters for biomass pyrolysis: A comparison of different lignocellulosic biomass. Energy Sources. Part A: Recovery, Utilization and Environmental Effects, 2019, vol. 41 (14), pp. 1690-1700.

45. Schwarzer L., Sárossy Z., Jensen P.A., Glarborg P., Karlström O., Holm J.K., Dam-Johansen K. Kinetic Parameters for Biomass under Self-Ignition Conditions: Low-Temperature Oxidation and Pyrolysis. Energy and Fuels, 2019, vol. 33 (9), pp. 8606-8619.

46. Alakangas E., Valtanen J., Levlin J.-E CEN technical specification for solid biofuels-Fuel specification and classes. Biomass and Bioenergy, 2006, vol. 30 (11), pp. 908-914.

47. Emeshev V.G., Parovinchak M.S. Bez privoznoy energetiki [No imported energy]. Neftegazovaya vertikal, 2005, no. 17, pp. 63-65.

48. Prilozhenie k Postanovleniyu Gosudarstvennoy Dumy Tomskoy oblasti ot 28.02.2008 no. 1008 «Energeticheskaya strategiya Tomskoy oblasti na period do 2020 goda» [Appendix to the Resolution of the State Duma of the Tomsk Region dated 02.28.2008 No. 1008 «Energy Strategy of the Tomsk Region for the Period Until 2020»]. Tomsk, The State Duma of the Tomsk Region, 2008. $89 \mathrm{p}$.

49. Inisheva L.I., Arkhipov V.S., Maslov S.G., Mikhanteva L.S. Torfyanye resursy Tomskoy oblasti $i$ ikh ispolzovanie [Peat resources of the Tomsk region and their use]. Novosibirsk, RASHN SB Publ., 1995. $88 \mathrm{p}$.

50. Vyazovkin S., Chrissafis K., Di Lorenzo M.L., Koga N., Pijolat M., Roduit B., Sbirrazzuoli N., Suñol J.J. ICTAC Kinetics Committee recommendations for collecting experimental thermal analysis data for kinetic computations. ThermochimicaActa, 2014, vol. 590, pp. 1-23.

51. Bercic G. The universality of Friedman's isoconversional analysis results in a model-less prediction of thermodegradation profiles. ThermochimicaActa, 2017, vol. 650, pp. 1-7.

52. Font R., Garrido M.A. Friedman and n-reaction order methods applied to pine needles and polyurethane thermal decompositions. ThermochimicaActa, 2018, vol. 660, pp. 124-133.

53. Havilah P.R., Sharma P.K., Gopinath M. Combustion characteristics and kinetic parameter estimation of Lantana camera by thermogravimetric analysis. Biofuels, 2019, vol. 10, pp. 365-372. 
54. IzatoY.-I., Miyake A. Kinetic analysis of the thermal decomposition of liquid ammonium nitrate based on thermal analysis and detailed reaction simulations. Journal of Thermal Analysis and Calorimetry, 2018, vol. 134, pp. 813-823.

55. Vassilev S.V., Vassileva C.G., Song Y., Li W., Feng J. Ash contents and ash-forming elements of biomass and their significance for solid biofuel combustion. Fuel, 2017, vol. 208, pp. 377-409.

56. Verma V.K., Bram S., Delattin F., Laha P., Vandendael I. Hubin A., De Ruyck J. Agro-pellets for domestic heating boilers: Standard laboratory and real life performance. Applied Energy, 2012, vol. 90, pp. 17-23.

57. DINplus pellets or ENplus pellets. Available at: https://www. biopelletmachine.com/biopellet-making-guidance/wood-pelletcertification-standard.html (accessed 25 February 2020).

58. Ibraeva K.T., Manaev U.O., Tabakaev R.B., Yazikov N.A., Zavorin A.S. Research of characteristics and mineral composition of peat of the Tomsk region relating to energy use. Bulletin of the Tomsk Polytechnic University. Geo Assets Engineering, 2019, vol. 330, no. 1, pp. 191-200. In Rus.

59. Kuznetsov B.N. Kataliticheskaya khimiya rastitelnoy biomassy [Catalytic chemistry of plant biomass]. Sorovskiy obrazovatelny zhurnal, 1996, no. 12, pp. 47-55.

60. Mohan D., Pittman Jr. C.U., Steele P.H. Pyrolysis of wood/biomass for bio-oil: a critical review. Energy and Fuels, 2006, vol. 20, pp. 848-889.

61. Simoneit B.R.T. Biomass burning - a review of organic tracers for smoke from incomplete combustion. Applied Geochemistry, 2002, vol. 17, pp. 129-162.

62. Zhao Y.L., Dolat A., Steinberger Y., Wang X., Osman A., Xie G.H. Biomass yield and changes in chemical composition of sweet sorghum cultivars grown for biofuel. FieldCropsResearch, 2009, vol. 111, pp. 55-64.

63. Sivenkov A.B. Vliyanie fiziko-khimicheskikh kharakteristik drevisiny na ee pozharnyu opasnost $i$ effektivnost ognezashchity. Avtoreferat Dokt. Dis. [Influence wood physicochemical characteristics on its fire hazard and fire protection effectiveness. Dr. Diss. Abstract]. Moscow, 2015. 49 p.

64. Peterson A.A., Vogel F., Lachance R.P., Froling M., Antal Jr. M.J., Tester J.W. Thermochemical biofuel production in hydrothermal media: A review of sub- and supercritical water technologies. Energy and Environmental Science, 2008, vol. 1, pp. 32-65.

65. Gronli M.G., Varhegyi G., Di Blasi C. Thermogravimetric analysis and devolatilization kinetics of wood. Industrial and Engineering Chemistry Research, 2002, vol. 41, pp. 4201-4208.

66. Kapaev G.I. Fiziko-khimicheskie osnovy protsessa termicheskogo razlozheniya soley ugolnoy kisloty. Avtoreferat Kand. Dis. [Physicochemical basis of thermal decomposition of carbonic acid salts. Cand. Diss. Abstract]. Moscow, 2009. 20 p.

67. Blasi C.D., Galgano A., Branca C. Exothermic events of nut shell and fruit stone pyrolysis. ACS Sustainable Chemistry and Engineering, 2019, vol. 7, pp. 9035-9049.

68. Tabakaev R.B., Astafev A.V., Dubinin Y.V., Yazykov N.A., Zavorin A.S., Yakovlev V.A. Autothermal pyrolysis of biomass due to intrinsic thermal decomposition effects. Journal of Thermal Analysis and Calorimetry, 2018, vol. 134, pp. 1045-1057.

69. Chen X., Zhang H., Xiao R. Mobile autothermal pyrolysis system for local biomass conversion: process simulation and techno-economic analysis. Energy and Fuels, 2018, vol. 32, pp. 4178-4188.

Received: 18 November 2020.

\section{Information about the authors}

Roman B. Tabakaev, Cand. Sc., researcher, National Research Tomsk Polytechnic University.

DarigaB. Altynbaeva, student, National Research Tomsk Polytechnic University.

Kanipa T. Ibraeva, postgraduate student, National Research Tomsk Polytechnic University.

Alexander S. Zavorin, Dr. Sc., professor, head of the dapartementNational Research Tomsk Polytechnic University. 